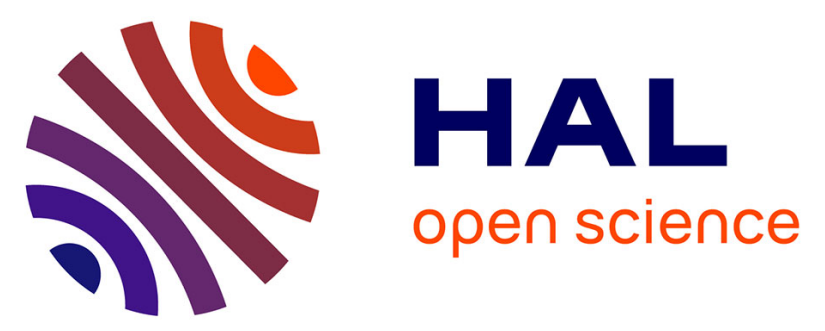

\title{
Regarder autrement le charbon de bois archéologique: l'exemple de l'irrigation des plantations d'oliviers en France méridionale et en Catalogne (IXe-XVe siècle).
}

Aline Durand, Jean-Frederic Terral

\section{- To cite this version:}

Aline Durand, Jean-Frederic Terral. Regarder autrement le charbon de bois archéologique: l'exemple de l'irrigation des plantations d'oliviers en France méridionale et en Catalogne (IXe-XVe siècle).. Archéologie du Midi Médiéval, 2005, La fructiculture. Cultures des fruits et lieux de culture de l'Antiquité, du Moyen Age et de l'Epoque moderne. Des savoirs en pratiques, des mots et des images, 23-24, pp.75-92. 10.3406/amime.2005.1825 . hal-01570869

\section{HAL Id: hal-01570869 \\ https://hal.science/hal-01570869}

Submitted on 1 Aug 2017

HAL is a multi-disciplinary open access archive for the deposit and dissemination of scientific research documents, whether they are published or not. The documents may come from teaching and research institutions in France or abroad, or from public or private research centers.
L'archive ouverte pluridisciplinaire HAL, est destinée au dépôt et à la diffusion de documents scientifiques de niveau recherche, publiés ou non, émanant des établissements d'enseignement et de recherche français ou étrangers, des laboratoires publics ou privés. 


\section{Regarder autrement le charbon de bois archéologique :}

l'exemple de l'irrigation des plantations d'oliviers en France méridionale et en Catalogne (IXe-XVe siècle).

Aline Durand, Jean-Frédéric Terral

\section{Citer ce document / Cite this document :}

Durand Aline, Terral Jean-Frédéric. Regarder autrement le charbon de bois archéologique : l'exemple de l'irrigation des plantations d'oliviers en France méridionale et en Catalogne (IXe-XVe siècle).. In: Archéologie du Midi médiéval. Tome 23-24, 2005. pp. 75-92;

doi : 10.3406/amime.2005.1825

http://www.persee.fr/doc/amime_0758-7708_2005_num_23_1_1825

Document généré le 14/04/2017 


\title{
Résumé
}

Pendant une trentaine d'années, le charbon de bois archéologique a plutôt été considéré par la communauté scientifique comme un objet strictement paléoenvironnemental, destiné à mesurer le poids de l'homme sur le milieu végétal. Les résultats anthracologiques contribuaient à écrire l'écohistoire des sociétés rurales et à développer l'archéologie des paysages passés. Pourtant, le charbon de bois enregistre aussi des pratiques agraires et des gestes techniques. Cette forme de lecture requiert la mise en oeuvre de protocoles inédits pour faire parler l'anatomie du bois en termes d'anthropologie historique. Grâce aux progrès de l'analyse d'images, l'éco-anatomie quantitative des bois, puis des charbons de bois, a pu se développer et autoriser ce type d'approches. Leur application à du matériel anthracologique archéologique, très récente, est faite ici sur des échantillons de charbons de bois médiévaux d'olivier. II s'agit de reconnaître pour la première fois l'existence d'une pratique, l'irrigation, en discriminant les individus à partir de signatures anatomiques référencées sur les bois et charbons de bois actuels et avec l'aide d'analyses factorielles. Cet article est une contribution méthodologique à l'histoire de l'arboriculture fruitière médiévale et change quelque peu le regard que l'on pouvait porter sur l'une des essences emblématiques de l'agriculture méditerranéenne.

\begin{abstract}
During the last thirty years, archaeological charcoal has been considered by the scientific community as a strictly paleoenvironmental object used to measure the impact of human activity on vegetal environment. Anthracological data helped writing the eco-history of rural societies and developing the archaeology of past landscapes. Yet, charcoal also records agrarian practices and technical gestures. Such reading requires using original protocols to question wood anatomy in terms of historical anthropology. Thanks to recent advances in picture analysis, the quantitative eco-anatomy of wood samples and later of charcoals was able to develop and allow such approach. Their application to archaeological anthracology is quite recent and, in the present case, on samples of medieval olive-tree charcoals. The aim is to detect for the first time an agricultural practice: irrigation, by discriminating individuals using referenced anatomical signatures on woods and charcoals of the present day and with the help of factorial analysis. This paper is a methodological contribution to the history of medieval fruit growing. It somewhat changes the way we see one of the emblematic species of trees of Mediterranean agriculture.
\end{abstract}




\title{
Des fruitiers en culture
}

\section{Regarder autrement le charbon de bois archéologique : l'exemple de l'irrigation des plantations d'oliviers en France méridionale et en Catalogne ( $\mathrm{IX}^{\mathrm{e}}-\mathrm{XV}^{\mathrm{e}}$ siècle).}

\begin{abstract}
Aline DURAND*, Jean-Frédéric TERRAL**
Pendant une trentaine d'années, le charbon de bois archéologique a plutôt été considéré par la communauté scientifique comme un objet strictement paléoenvironnemental, destiné à mesurer le poids de l'homme sur le milieu végétal. Les résultats anthracologiques contribuaient à écrire l'éco-histoire des sociétés rurales et à développer l'archéologie des paysages passés. Pourtant, le charbon de bois enregistre aussi des pratiques agraires et des gestes techniques. Cette forme de lecture requiert la mise en œuvre de protocoles inédits pour faire parler l'anatomie du bois en termes d'anthropologie historique. Grâce aux progrès de l'analyse d'images, l'éco-anatomie quantitative des bois, puis des charbons de bois, a pu se développer et autoriser ce type d'approches. Leur application à du matériel anthracologique archéologique, très récente, est faite ici sur des échantillons de charbons de bois médiévaux d'olivier. Il s'agit de reconnaître pour la première fois l'existence d'une pratique, l'irrigation, en discriminant les individus à partir de signatures anatomiques référencées sur les bois et charbons de bois actuels et avec l'aide d'analyses factorielles. Cet article est une contribution méthodologique à l'histoire de l'arboriculture fruitière médiévale et change quelque peu le regard que l'on pouvait porter sur l'une des essences emblématiques de l'agriculture méditerranéenne.

During the last thirty years, archaeological charcoal has been considered by the scientific community as a strictly paleoenvironmental object used to measure the impact of human activity on vegetal environment. Anthracological data helped writing the eco-history of rural societies and developing the archaeology of past landscapes. Yet, charcoal also records agrarian practices and technical gestures. Such reading requires using original protocols to question wood anatomy in terms of historical anthropology. Thanks to recent advances in picture analysis, the quantitative eco-anatomy of wood samples and later of charcoals was able to develop and allow such approach. Their application to archaeological anthracology is quite recent and, in the present case, on samples of medieval olive-tree charcoals. The aim is to detect for the first time an agricultural practice : irrigation, by discriminating individuals using referenced anatomical signatures on woods and charcoals of the present day and with the help of factorial analysis. This paper is a methodological contribution to the history of medieval fruit growing. It somewhat changes the way we see one of the emblematic species of trees of Mediterranean agriculture.
\end{abstract}

Mots-clés : anthracologie, éco-anatomie quatitative, épistémologie, historiographie, irrigation, olivier, Méditerranée nord-occidentale, terroir.

Key words : historiography, epistemology, olive tree, charcoal analysis, quantitative eco-anatomy, irrigation, terroir, North-West Mediterranean area.

\footnotetext{
* MCF, Département d'Histoire et LAMM UMR 6572, Université de Provence Aix-Marseille I, 29 avenue R. Schuman 13621 Aix-en-Provence cédex 1. adurand@mmsh.univ-aix.fr

** MCF, Département de biologie, Université des Sciences et Techniques du Languedoc (Montpellier II) et UMR 5059 Centre de Bioarchéologie et d'Ecologie (CBAE), Institut Botanique, 163 rue Auguste Broussonnet 34090 Montpellier, terral@ crit.univ-montp2.fr
} 
Le charbon de bois est l'un des matériaux, si ce n'est le matériau, le plus abondant sur les sites archéologiques. Même quand le mobilier céramique, métallique... est absent, les structures arasées ou à peine lisibles, l'archéologue du fugace sait qu'il peut compter sur les petits bouts de bois brûlés pour lui fournir une datation. Depuis la découverte de la méthode radiocarbone, c'est d'abord sous cet angle que les vestiges ligneux carbonisés ont intéressé la communauté scientifique. Et pendant plus d'un demi-siècle, les charbons de bois ont uniquement été des auxiliaires précieux pour établir la chronologie des évolutions sociales depuis la conquête prométhéenne du feu établie autour de 400000 ans avant notre ère à Terra Amata.

C'est dans les années cinquante-soixante que les charbons de bois suscitent l'intérêt des paléobotanistes. Certes, depuis la fin du $\mathrm{XIX}^{\mathrm{e}}$ siècle, quelques études ponctuelles avaient bien été menées en Suisse ou en Allemagne, en France par l'abbé H. Breuil, ou ailleurs, pour déterminer les bois ayant alimenté les foyers, mais sans perspective paléofloristique d'ampleur. Ce n'est pas le cas du hongrois J. Stieber $(1967,1969)$ qui, le premier, en 1967, fait sortir le charbon de bois de l'anonymat archéologique en lui conférant une dimension large, autre que chronologique. À partir de cette date, les analyses se sont multipliées d'abord dans une perspective strictement qualitative : regarder l'évolution de la flore. Puis, avec le développement de l'observation en lumière réfléchie, les analyses quantitatives ont pris leur essor, érigeant le charbon de bois archéologique au rang de source paléoécologique à part entière (1), au même titre que la palynologie qui, sur ce plan, a gagné ses lettres de noblesse depuis presque un siècle.

Cette avancée technique a donc ouvert la voie à l'archéologie des paysages et à l'éco-histoire. Les petits bouts de bois carbonisés ont alors été regardés comme des objets paléoenvironnementaux. L'archéologue ou l'historien se sont appuyés sur leur témoignage pour écrire l'histoire des communautés phytogéographiques, du climat, de la sociabilisation, de l'artificialisation, ou, pour employer un terme au contenu très chargé et qui mériterait à lui seul un commentaire, de l'anthropisation du milieu naturel (Thiébault 1988 ; Chabal, 1997 ; Durand, 1998). Cette évolution a été rendue lisible et officielle. En effet, le paléoenvironnement est devenu une matière obligatoire dans la formation universitaire des archéologues. Pour qui a suivi l'évolution de la discipline depuis vingt ans, ce constat ne peut que réjouir. D'abord parce qu'il contribue à élargir sans ambiguiité la définition du site archéologique à ce que l'on appelait encore il n'y a pas si longtemps le « hors site ». Ensuite parce que l'étude de l'homme social n'est plus le cœur unique de la conception actuelle de l'archéologie et de l'histoire. Depuis quelques années, l'étude des charbons de bois archéologiques s'est uniquement développée dans cette direction. Et l'on assiste à une forme de cantonnement, voire de normalisation, mais le terme est sans doute outrancier, de la discipline à un seul type d'études et de résultats. Cette situation découle de la difficulté initiale à faire admettre comme un matériau archéologique à part entière le matériel bioarchéologique, charbons de bois, graines ou pollens, et, également, à les faire considérer comme une véritable source historique au même titre que les parchemins. Et, de fait, jusqu'à présent, la dimension sociale et culturelle de l'objet d'étude a été oubliée, voire même quelquefois déniée.

Cependant, le charbon de bois est de plus en plus regardé aussi comme le rebut de l'activité domestique faite par l'homme : la maîtrise et la pratique du feu sont aussi des actes sociaux qu'il faudra un jour analyser comme tels pour les périodes historiques, car cette voie d'approche a déjà été empruntée pour des sociétés beaucoup plus anciennes (Perlès 1977). La naissance de nouveaux moyens techniques, conjuguée à une appréhension plus complexe de la relation entre une société et son milieu, rendent désormais possibles de telles études. Il faut également garder cette dimension culturelle pour le matériau lui-même. Le charbon de bois est la boîte noire de l'environnement passé : il enregistre fidèlement les fluctuations de la couverture végétale, mesure l'impact d'une société humaine sur le paysage, révèle la dynamique des communautés végétales... Mais il a aussi enregistré des pratiques agraires et des gestes techniques. Cette forme de lecture requiert la mise en œuvre de protocoles inédits pour faire parler l'anatomie du bois en termes d'anthropologie historique. Mais avant de les développer, puis de montrer leur application archéologique, ici à la culture des fruitiers et participer ainsi à écrire l'histoire de l'arboriculture fruitière, il faut prendre la mesure du courant historiographique qui a porté leur naissance.

\section{HISTORIOGRAPHIE D'UNE MÉTHODOLOGIE NOUVELLE}

L'étude anthropologique de l'homme préhistorique a constitué l'un des moteurs essentiels du développement de l'archéologie. L'un de ses principaux résultats est d'avoir sociabilisé la très longue durée en découpant le temps au rythme d'une évolution biologique de l'homme fossile. Il s'agissait avant tout de suivre la genèse progressive de l'homme social. Dans cette perspective, il apparaissait, et il apparaît encore naturel, aux yeux des praticiens de la discipline, de suivre aussi la naissance d'activités proprement sociales, comme autant de marqueurs forts de la transformation de l'environnement par l'homme. Saisir le processus complexe de la domestication de la flore et de la faune a porté le développement des études bioarchéologiques de morphométrie et d'éco-anatomie quantitative sur le matériel biologique livré par les fouilles. 


\section{Naissance et intérêt d'études morphométriques}

Depuis le $\mathrm{XIX}^{\mathrm{e}}$ siècle, les archéobotanistes ont donc cherché des outils pour mesurer le plus finement possible la diffusion de l'agriculture. Bien évidemment ce sont les céréales qui ont retenu d'abord et avant tout leur attention. Mais ils n'ont pas pour autant délaissé les arbres fruitiers : l'olivier, la vigne, le figuier, le dattier ont suscité plusieurs travaux relatifs à leur domestication et la diffusion de leurs cultivars tout autour du Bassin méditerranéen (Chevalier 1948 ; Zohary et Spiegel-Roy 1975 ; Zohary et Hopf 1988). Ils ont contribué à étayer le modèle diffusionniste de la néolithisation de l'Orient vers l'Occident. Progressivement, les chercheurs ont commencé aussi à se pencher sur un matériel plus récent et plus occidental : les nombreux noyaux de fruits de Prunoïdées, famille botanique qui rassemble la plupart des arbres à noyaux cultivés des vergers actuels (pruniers, cerisiers, pêchers, amandiers, abricotiers...) découverts dans les niveaux archéologiques protohistoriques, antiques, puis plus récemment médiévaux, ont favorisé l'émergence d'études biométriques. L'école archéobotanique anglaise, et surtout, néerlandaise et allemande, s'est préoccupée, depuis le milieu du $\mathrm{XX}^{\mathrm{e}}$ siècle, d'analyser la forme des noyaux («Formenkreise») et leurs dimensions (biométrie). Mais la perspective demeurait toujours une perspective diffusionniste : cerner l'apparition d'une essence fruitière révélatrice de l'impact d'une société, de son patrimoine botanique et technique (Bertsch 1941 ; Baas 1974 ; Renfrew 1973 ; Behre 1978, 1981). En l'occurrence, il s'est souvent agi de mesurer l'influence du monde grec en Occident ou la réalité de la Romanisation en Europe.

Parmi les fruitiers, c'est évidemment la vigne qui a fait l'objet de toutes les attentions. Pour discriminer la vigne sauvage de la vigne cultivée, dès le début du siècle, des critères biométriques basés sur le rapport longueurlargeur des pépins ont été mis au point (Stummer 1911). Cet indice a très longtemps été utilisé par les carpologues. En France méridionale, Ph. Marinval (1988) en a réalisé la première exploitation d'envergure pour établir la date d'apparition de la viticulture au second Âge du Fer (Marinval 1988). Actuellement, la communauté scientifique juge insuffisant la prise en compte de ce seul indice et s'efforce d'en mettre au point de plus idoines (Di Vora et Castelletti 1995 ; Mangafa et Kotsakis 1996 ; Marinval 1997). Pour distinguer les deux sous-espèces de vigne, cultivée et sauvage, l'approche morphométrique s'est révélée pertinente et a confirmé l'existence de cultivars dès le $\mathrm{V}^{\mathrm{e}}$ siècle avant notre ère (Bouby et Marinval 2001).

Les carpologues septentrionaux quant à eux, ont cherché à construire des protocoles d'études biométriques sur les Rosacées, et plus particulièrement sur les pruniers, car les noyaux de prunelles sont parmi les carporestes fruitiers les plus abondants. Ils ont ainsi proposé une différenciation variétale reposant sur des critères morphologiques et biométriques (Röder 1940 ; Bertsch 1941 ; Baas 1974 ; Behre 1978 ; et voir bibliographie du présent volume) et fait avancer le débat sur le statut réel de certains fruitiers comme Prunus spinosa, le prunellier. Mais là encore les critères n'ont pas été jugés totalement satisfaisants.

C'est pourquoi, actuellement, les bioarchéologues développent un nouveau type d'approche biométrique et morphologique : la morphométrie géométrique. Elle regroupe un certain nombre de méthodes visant à des descriptions rigoureuses de la morphologie des échantillons par l'analyse de leur structuration géométrique. Ces méthodes permettent non seulement une étude conjointe de la «taille » et de la géométrie des objets, mais également de la «forme», et ce, indépendamment de la taille, ce qui est d'un intérêt majeur. Cette analyse revient, en effet, à étudier le déterminisme génétique de variabilité génétique. Cette méthode est actuellement testée sur la vigne après l'avoir été sur l'olivier (Terral 1997a et b). On en trouvera une belle illustration dans le présent volume (Bouby et al.).

\section{Le développement de l'éco-anatomie quantitative sur le bois et les charbons de bois}

Tout naturellement, ces orientations récentes en matière de morphométrie et de biométrie se sont progressivement appliquées au matériel ligneux, et non plus seulement au seul matériel carpologique. Ainsi, sous l'impulsion de J.-F. Terral (1996, 1997a et b, 2000, 2004), les études d'éco-anatomie quantitative du bois se sont développées. Leur principe réside en la mesure des éléments vasculaires du bois et de leurs variations. En effet, au cours d'un cycle annuel de végétation, période pendant laquelle le cerne ou anneau de croissance est édifié, la mise en place des éléments structuraux du bois est modulée sous l'effet des actions conjuguées du climat et d'autres facteurs de variations, intrinsèques ou extrinsèques, accidentels ou non : l'âge, les conditions édaphiques, l'altitude, l'exposition, les facteurs anthropiques (pratiques culturales et traitements sylvicoles), les agents pathogènes ainsi que les facteurs polluants. Tout au long de sa vie, l'arbre s'adapte aux éventuelles variations de son milieu. L'anatomie du bois est alors influencée par pratiquement tous les événements qui interviennent dans la croissance et le développement de l'arbre. Parmi les facteurs qui expriment cette dépendance de l'anatomie du bois vis-àvis du milieu, le climat et les activités humaines sont prépondérants.

Au cours de son développement, l'arbre est soumis à un ensemble de forces (poids des branches, vent par exemple) qui détermine son état de «contrainte mécanique ». L'arbre réagit à ces contraintes en produisant un bois que l'on qualifie de bois de réaction. Les cellules du bois formées chaque année prennent leur structure définitive et subissent la maturation cellulaire ou phase de lignification. Au cours de cette phase de maturation, les cellules ont tendance à se déformer. Ces déformations sont appelées «déformations de maturation » (Fig. 1). Ces phénomènes sont observés dans le bois émis par le tronc, mais surtout dans le bois 


\section{Le processus de maturation du bois}

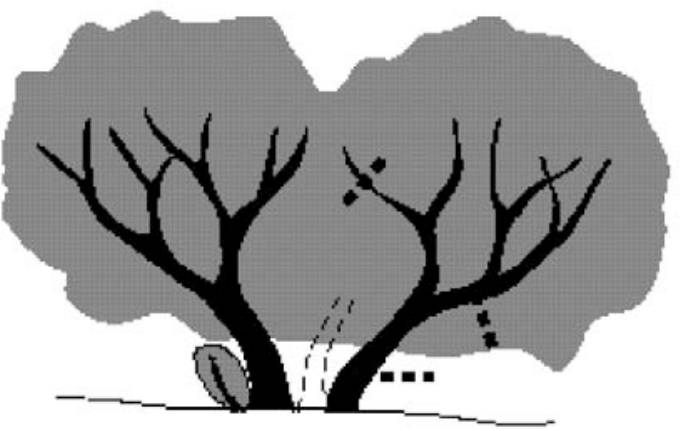

-.... Echantillon de bois

\section{Echantillons de branches jeunes}

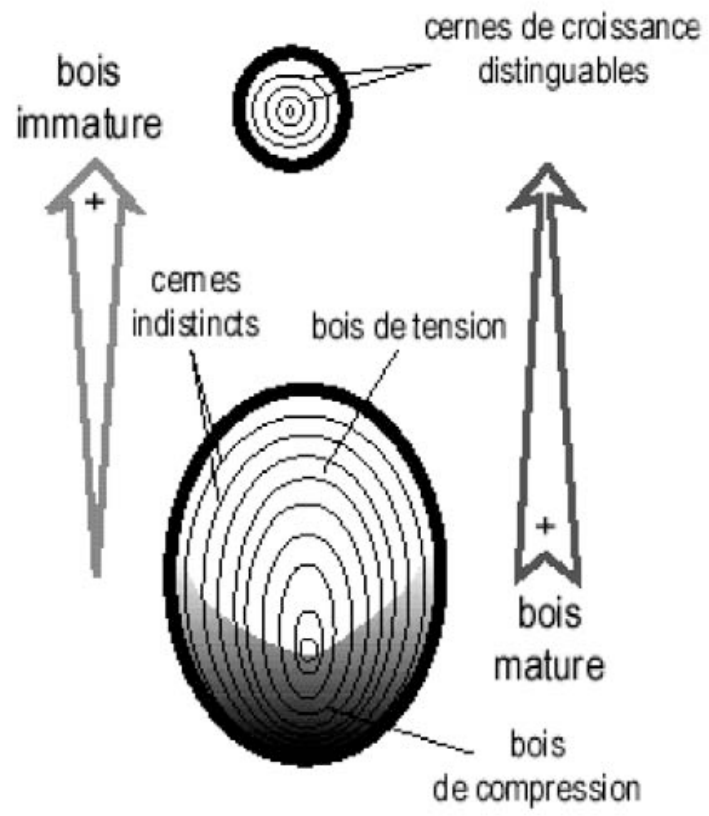

Echantillons de branches charpentières et de trono
Fig. 1 : Le processus de maturation du bois. Dessin J.-F. Terral.

de branches qui ont une direction de croissance oblique ou horizontale. C'est à partir de ces déformations que peut être tentée la discrimination des bois entre les bois provenant de branches ou de troncs. Sur des échantillons archéologiques, des pratiques telles que la taille et l'émondage pourront alors être mises en évidence.

Pour passer de la théorie à la pratique, les xylologues et les anthracologues ne pouvaient jusqu'à présent compter que sur l'observation microscopique à l'aide d'un micromètre. Ils s'y sont essayés à plusieurs reprises, notamment sur le chêne à feuillage caduc méditerranéen et l'olivier pour différencier le sauvage du cultivé ou l'espèce à déterminer. Les résultats étaient contrastés (Vernet et al. 1987 ; Badal Garcia 1984 ; Grau Almero 1984 ; Solari, 1988). La précision des mesures obtenues n'était pas satisfaisante. Ce sont les progrès des techniques et des méthodes de l'analyse d'images qui ont rendu possible la caractérisation de signatures fiables avec l'assistance de l'éco-anatomie quantitative (QEA) (Terral et Arnold-Simard 1996). L'analyse à haute résolution du bois et de la structure du charbon de bois est devenue réalisable avec une marge d'erreur statistique infime. La recherche des signatures anatomiques de pratiques, comme l'irrigation ou l'amendement, pouvait être entreprise. Car l'analyse anatomique quantitative discrimine les espèces en fonction de leur statut écologique, c'est-à-dire en fonction de paramètres environnementaux, et non génétiques.

Cette évolution, très récente, de la recherche bioarchéologique, et anthracologique en particulier, réconcilie le charbon de bois avec une dimension sociale et historique jusque là quelque peu négligée. Ces nouveaux outils font des échantillons carbonisés, non plus seulement des objets paléoécologiques, mais aussi des objets culturels à part entière, au sens premier du terme. Ils renouent fermement le fil entre l'écofact et l'artéfact et tissent la trame d'une archéologie des savoirs et des savoir-faire, socle à partir duquel se construit la compréhension historique des terroirs et des paysages.

\section{DOCUMENTER UNE PRATIQUE PAR UNE APPROCHE ÉCO-ANATOMIQUE : PROTOCOLE D'ÉTUDE POUR UNE ARCHÉOLOGIE DES GESTES DE LA FRUCTICULTURE}

Appliquer très concrètement cette démarche pour circonscrire un geste technique passe obligatoirement par le choix d'une essence sur laquelle développer un tel regard. En domaine méditerranéen, le choix de l'olivier s'impose pour mettre en œuvre un tel programme de 
recherche. La forte charge symbolique de l'arbre, son importance réelle, fictive ou rêvée, en tout cas débattue chez les historiens comme chez les archéologues, les antiquisants comme les médiévistes (Amouretti et Comet, 1985-2000, Laval et al. 1990, Boyer 1991, Leveau et al. 1991, Comet 2001, 2002, Amouretti et Brun 2002) ont été de solides arguments pour ouvrir le dossier archéologique et historique de l'arboriculture fruitière aux époques historiques et particulièrement durant le Moyen Âge. Le travail parallèle mené par les biologistes et les agronomes sur l'anatomie de ce bois en a été un autre. Il fallait donc réunir les compétences et croiser les méthodologies. L'Aide à Projet Nouveau du CNRS coordonnée par A. Durand (2001-2003), puis l'ACI Fructiculture du programme Histoire des Savoirs du CNRS coordonnée par M.-P. Ruas (2003-2006) en ont grandement facilité la réalisation.

L'olivier est fréquemment présenté dans la littérature historique comme un arbre rustique que l'on plante dans les terres rocailleuses, sèches, étagées en terrasses des collines et des montagnes méridionales (Amir 1998). L'arbre ne nécessiterait aucun soin, surtout pas un apport d'eau. Mais cette image très traditionnelle et figée de l'arbre correspond-elle à la réalité médiévale ? Il fallait chercher à le vérifier en documentant anthracologiquement la pratique de l'irrigation, si importante dans la conduite arboriculturale de l'arbre et dans l'appréciation portée sur le type de terroir d'olivettes. C'est uniquement sous l'angle méthodologique que cette vérification sera ici tentée car la démarche, en termes de biologie et d'éco-anatomie, a déjà été exposée ailleurs (Terral et Durand 2006) ; la discussion sur la signification des résultats en termes d'archéologie et d'histoire des savoirs arboriculturaux et leur confrontation avec les données archéologiques et historiques également (Durand 2004). À travers l'exemple de l'olivier, les compétences se sont rejointes. Ici, le parti-pris méthodologique, historiographique et épistémologique constitue la pierre angulaire d'un discours anthropologique sur les pratiques de l'arboriculture fruitière au Moyen Âge, comme aux autres époques.

\section{Établir un protocole référencé sur l'actuel}

La première étape d'une telle recherche consiste à établir un protocole de référence sur l'actuel des variations anatomiques signifiantes $\mathrm{du}$ facteur environnemental recherché, en l'occurrence l'irrigation. Pour former le corpus de référence, les bois d'Olea europaea ont été collectés par J.-F. Terral (1997a et 2000) dans 20 sites de l'ouest du bassin méditerranéen, à l'exception de la Grèce (fig. 2). L'attention s'est particulièrement concentrée sur le Levant, une région de l'Espagne, très contrastée d'un point de vue écologique. C'est la raison pour laquelle pas moins de sept stations y ont été définies. Pour prendre en compte la variabilité interne de chaque individu, les prélèvements ont été effectués à différentes hauteurs du sujet. Le protocole de référence comprend 446 échantillons : 241 de stations d'oliviers sauvages en conditions naturelles, 45 de stations d'oliviers sauvages en conditions de ripisylve, 114 de stations d'oliviers cultivés à sec et 46 d'oliviers cultivés irrigués. Dans ce dernier cas, l'irrigation pratiquée était une irrigation traditionnelle, par des techniques d'irrigation gravitaire, et non pas modernes, comme par aspersion. Le terme gravitaire s'applique à des systèmes où l'eau circule principalement dans des

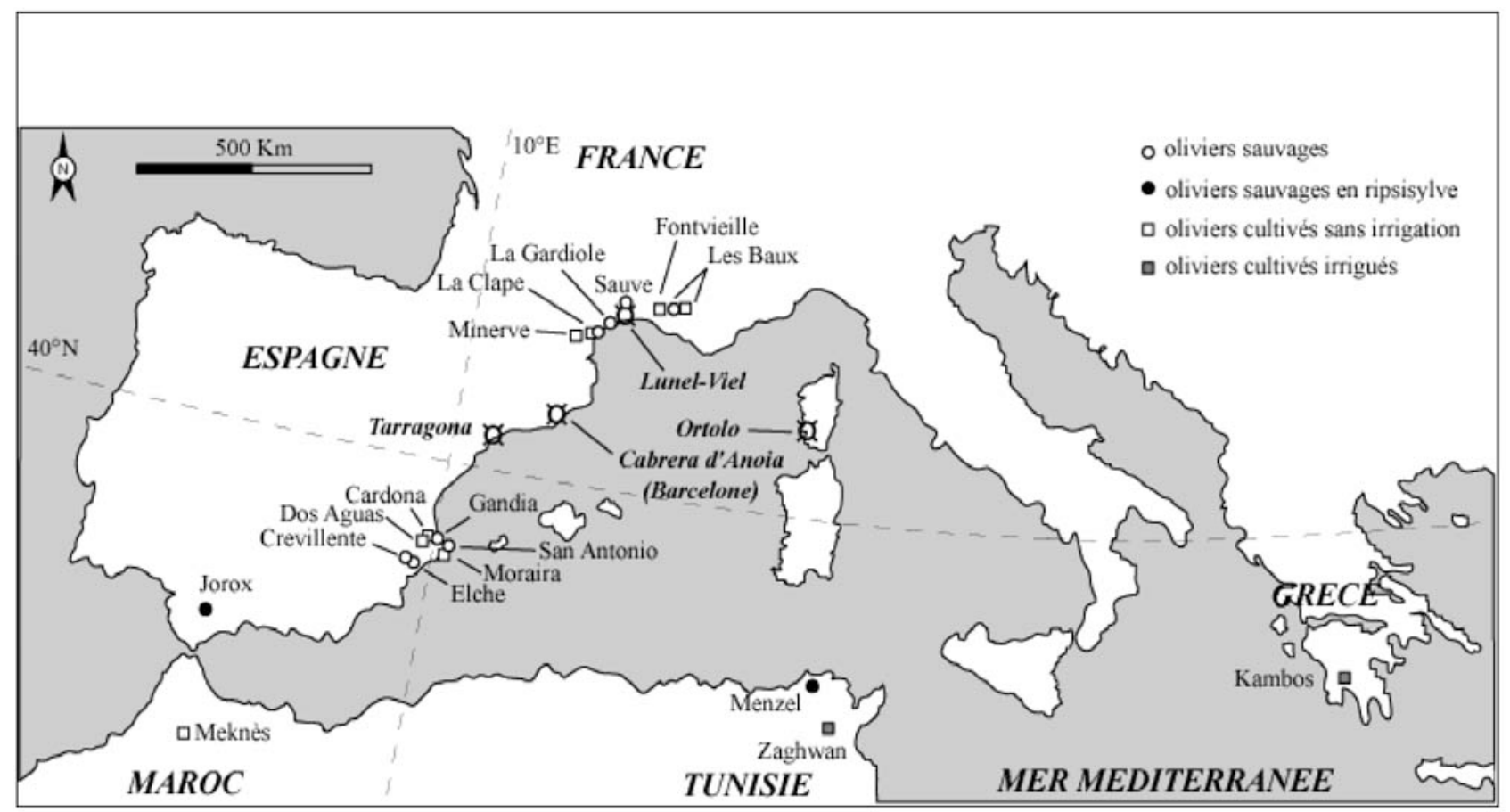

Fig. 2 : Localisation des populations d'où sont issus les échantillons de référence et localisation des sites archéologiques médiévaux étudiés. Carte J.-F. Terral. 
canaux à écoulement libre. Les échantillons ont été ensuite séchés et carbonisés dans un four électrique en milieu anaérobie à $400-450^{\circ} \mathrm{C}$.

Comme tous les fruitiers, l'olivier réagit favorablement à tout apport extérieur régulier favorisant sa croissance. Dans le cas de l'irrigation, il est logique de penser que les éléments anatomiques de conductivité hydraulique, les vaisseaux, vont être plus nombreux et plus importants en taille afin de faciliter les échanges et la circulation de l'eau et des sels minéraux dans le cas dans d'un sujet bénéficiant d'un apport d'eau que dans le cas d'un sujet cultivé à sec. Il est également logique penser que l'accroissement annuel des cernes sera en moyenne plus important dans le cas d'un arbre profitant habituellement de soins que dans le cas d'un arbre laissé à vau-l'eau. Par conséquent, en coupe transversale, une batterie de tests statistiques a été élaborée le long d'un transect dans le sens de la croissance du bois et en traversant plusieurs cernes de croissance afin de cerner les paramètres anatomiques discriminants (fig. 3).

Pour signer anatomiquement la réalité de l'irrigation sur un bois d'olivier, le protocole mis au point prend en compte quatre critères biométriques (Terral 1997a) : la longueur du cerne de croissance (GWR, $\mu \mathrm{m})$, la surface des vaisseaux (SVS, $\mu \mathrm{m}^{2}$ ), la densité des vaisseaux (DVS, N/mm²), le nombre de vaisseaux par groupes (NVS). Pour obtenir des résultats statistiquement fiables, plusieurs mesures par critères sont nécessaires : pour le diamètre des vaisseaux, une bonne trentaine par échantillon au minimum. La conductivité hydraulique

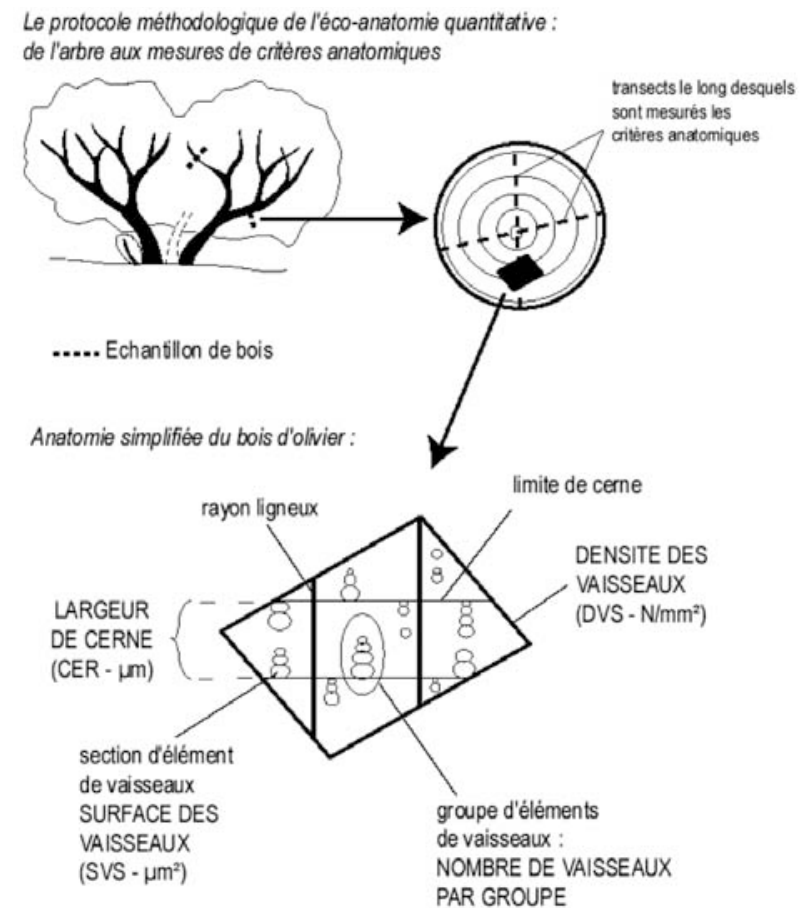

COEFFICIENT DE CONDUCTIVITE

HYDRAULIQUE :

$\mathrm{CD}=(\mathrm{SVS} / \pi)^{2} / \mathrm{DVS}$

Fig. 3 : Le protocole méthodologique de l'éco-anatomie quantitative.

Dessin J.-F. Terral. des vaisseaux $\left(C D=[\text { Surface des vaisseaux } / \pi]^{2} /\right.$ densité des vaisseaux]) est également calculée (Zimmermann 1983 ; Carlquist 1988). D'un point de vue pratique, les mesures s'effectuent sur un microscope à réflexion couplé à un analyseur d'images. Pour la présente étude, elles ont toutes été réalisées sur le matériel de la station du Centre de bio-archéologie et d'écologie de l'université des sciences et techniques du Languedoc à Montpellier implanté à l'Institut de botanique.

Les données quantitatives éco-anatomiques sont ensuite traitées par deux analyses factorielles discriminantes (AFD). Associées à des analyses statistiques multivariées, les données mesurées se voient appliquer le test non paramétrique de Mann-Whitney (Utest) afin d'évaluer la réponse du bois à un apport extrinsèque d'eau sur les échantillons d'oliviers sauvages et d'oliviers cultivés. Le choix de ce test plutôt qu'une analyse de variance (ANOVA) est justifié par le fait que les groupes d'oliviers sont constitués d'échantillons de populations distinctes. Dans ce cas, le test (U-test) ne nécessite pas une distribution normale, autrement dit gaussienne, des données (Sokal et Rohlf 2000).

La première AFD est menée sur 446 échantillons de bois brûlés issus des populations contemporaines de référence à l'aide de six variables : cinq variables quantitatives éco-anatomiques et une variable qualitative exprimant les modalités de correspondances des quatre groupes d'oliviers. Cette analyse cherche à mettre en évidence des critères fiables discriminants entre les différentes populations et à établir le lien entre lesdits critères et lesdits groupes. La seconde AFD concerne elle aussi les 446 échantillons de référence contemporains et prend en compte les cinq variables éco-anatomiques quantitatives, à l'exception du caractère «longueur du cerne de croissance », et la variable qualitative définissant les quatre groupes d'oliviers.

\section{Les résultats de l'analyse éco-anatomique et statistique : le modèle de référence}

Les données quantitatives contemporaines traitées par les deux AFD sont synthétisées dans le tableau cidessous (tabl. 1).

Elles sont également représentées graphiquement (fig. 4). Le pouvoir discriminant calculé par la première AFD est égal à 93,4 \%. Le premier axe canonique (Axe 1) de cette AFD fondée sur l'ensemble des caractères anatomiques, exprimant $57,2 \%$ de la variance totale, discrimine les oliviers croissant sous influence hydrique des oliviers se développant en conditions de sécheresse. Les caractères contribuant à la discrimination de ces deux groupes sont la surface des vaisseaux et la conductivité vasculaire, deux variables fortement corrélées. L'axe canonique $2(32,2 \%$ de la variance totale) distingue les oliviers sauvages poussant en conditions naturelles (en sec) et caractérisés par des cernes de croissance étroits, des oliviers sauvages de ripisylve. 


\begin{tabular}{|c|c|c|c|c|}
\hline $\begin{array}{l}\text { Population } \\
\text { d'olivier }\end{array}$ & $\begin{array}{l}\text { Nombre } \\
\text { d'échantillons }\end{array}$ & $\begin{array}{l}\text { Critère } \\
\text { anatomique }\end{array}$ & Moyenne & Écart-type \\
\hline Sauvage à sec & 241 & $\begin{array}{l}\text { GRW } \\
\text { SVS } \\
\text { NVS } \\
\text { DVS } \\
\text { CD }\end{array}$ & $\begin{array}{l}762.45 \\
592.21 \\
2.03 \\
188.37 \\
236.22 \\
\end{array}$ & $\begin{array}{l}118.57 \\
131.37 \\
0.18 \\
66.06 \\
154.87 \\
\end{array}$ \\
\hline Sauvage en ripisylve & 45 & $\begin{array}{l}\text { GRW } \\
\text { SVS } \\
\text { NVS } \\
\text { DVS } \\
\text { CD }\end{array}$ & $\begin{array}{l}786.45 \\
1165.12 \\
2.12 \\
221.57 \\
632.16\end{array}$ & $\begin{array}{l}70.27 \\
125.69 \\
0.12 \\
15 \\
148.70\end{array}$ \\
\hline Cultivé à sec & 114 & $\begin{array}{l}\text { GRW } \\
\text { SVS } \\
\text { NVS } \\
\text { DVS } \\
\text { CD }\end{array}$ & $\begin{array}{l}1084.31 \\
605.15 \\
1.87 \\
144.95 \\
268.22\end{array}$ & $\begin{array}{l}99.29 \\
120.35 \\
0.17 \\
26.70 \\
99.44\end{array}$ \\
\hline Cultivé irrigué & 46 & $\begin{array}{l}\text { GRW } \\
\text { SVS } \\
\text { NVS } \\
\text { DVS } \\
\text { CD }\end{array}$ & $\begin{array}{l}1131.23 \\
1088.14 \\
1.83 \\
162.29 \\
757.74\end{array}$ & $\begin{array}{l}99.29 \\
128.67 \\
0.08 \\
19.58 \\
203.27\end{array}$ \\
\hline
\end{tabular}

Tab. 1 : Synthèse des données quantitatives (Moyenne et écart-type pour chaque population d'oliviers).

La seconde AFD, dans laquelle le caractère anatomique «largeur de cerne» n'a pas été inclus, discrimine $76,1 \%$ des échantillons d'olivier. Même si le taux de discrimination de cette seconde AFD est plus faible que celui de la première, il apparait que 3 groupes se différencient parfaitement. L'axe canonique 1 (81,9\% de la variance) discrimine, quel que soit le statut sauvage ou cultivé, les oliviers poussant sous influence hydrique des oliviers en conditions de sécheresse. L'axe canonique 2 (13,1\% de la variance) distingue les oliviers sauvages de ripisylve des oliviers cultivés et irrigués.

D'autres résultats non démontrés par les AFD ont été mis en évidence par le test non paramétrique de MannWithney. Bien que les performances de ce test ne soient plus à démontrer (Sokal et Rohlf 2000), par choix, seuls les résultats les plus significatifs ont été discutés. Ainsi, des différences de nombre de vaisseaux par groupe (NVS) ont été détectées en relation avec la disponibilité de l'eau pour l'arbre (tab. 2). Pour les oliviers sauvages, NVS est sensiblement plus élevé chez les arbres poussant en conditions sèches que chez les arbres de ripisylve. De plus, une augmentation de densité de vaisseaux est notée depuis les oliviers cultivés en sec vers les oliviers irrigués.

\section{Signification écologique des résultats éco-anatomiques}

L'olivier sauvage est particulièrement bien adapté au climat méditerranéen caractérisé notamment par des conditions thermiques et hydriques estivales très stressantes pour les végétaux. L'olivier sauvage pousse généralement sur des sols bien drainés, calcaires, et il est une pièce maîtresse des matorrals du bassin méditerranéen. Dans les zones semi-arides comme dans le sud de l'Espagne et en Afrique du nord, les oléastres peuvent être rencontrés sur les rives de cours d'eau temporaires (oueds dans le Maghreb ou barrancos dans la péninsule ibérique). Cette situation écologique atypique offre aux arbres un apport d'eau leur permettant, au printemps, de se développer dans des conditions optimales. L'été, les cours d'eau sont totalement asséchés et la croissance des arbres est stoppée.

Les oliviers mis en culture croissent plus rapidement que leurs congénères sauvages (Terral et Arnold-Simard 1996). Même s'ils sont cultivés à sec, un espacement des individus, l'élimination des compétiteurs ou des

\begin{tabular}{lccc} 
Comparaison & $\begin{array}{c}\text { Critère } \\
\text { anatomique }\end{array}$ & U-test & P \\
\hline Sauvage à sec x Sauvage en ripisylve & CER & 4656 & 0.13 \\
& SVS & 7 & $<0.001^{* *}$ \\
& NVS & 3216 & $<0.001^{* *}$ \\
& DVS & 295 & $0.03^{*}$ \\
& CD & 386 & $<0.001^{* *}$ \\
\hline Cultivé à sec x Cultivé irrigué & CER & 1887.5 & $0.01^{*}$ \\
& SVS & 13 & $<0.001^{* *}$ \\
& NVS & 2081 & $0.04^{*}$ \\
& DVS & 1355.5 & $<0.001^{* *}$ \\
& CD & 26 & $<0.001^{* *}$ \\
\hline significatif - **: hautement significatif & \multicolumn{3}{c}{}
\end{tabular}

Tab. 2 : Résultats du test non paramétrique de Mann-Whitney pour tester l'effet d'un apport en eau sur l'anatomie des bois d'oliviers sauvages et cultivés. (Test J.-F. Terral). 




Fig. 4 : Représentation graphique des résultats des analyses factorielles discriminantes

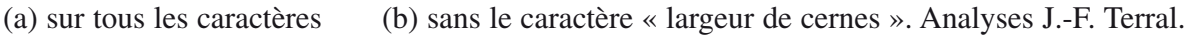


amendements suffisent à expliquer des différences de cinétique de croissance. Néanmoins, les régions sèches et semi-arides dépendent de l'irrigation en vue de garantir une production agricole suffisante (Herrero et Snyder 1997). Dans d'autres régions, où les conditions climatiques sont moins drastiques, l'irrigation peut être tout de même employée dans le but d'augmenter la productivité, le rendement et la qualité des récoltes (Patumi et al. 2002)

D'un point de vue biologique, une relation significative entre les caractéristiques anatomiques et la disponibilité de l'eau dans le milieu a été mise en évidence, à la fois chez l'olivier sauvage et chez l'olivier cultivé. La conductivité hydraulique, liée directement au caractère "surface des vaisseaux » augmente fortement en réponse à un apport d'eau. Une surface de vaisseaux élevée et donc de conductivité, peut être interprétée comme une adaptation et une réponse éco-physiologique de l'anatomie du bois. Ces changements correspondent donc à une grande efficacité de transport de sève.

Étayant ces résultats, Wimmer et al. (2002) ont montré qu'un apport hydrique stimule le fonctionnement cambial. Toutefois, l'augmentation de croissance radiale mise en évidence chez les oliviers cultivés et irrigués par rapport aux oliviers cultivés à sec, n'est pas constatée chez l'olivier sauvage. Chez ces derniers, les caractères «nombre de vaisseaux par groupe » et «densité de vaisseaux » ne semblent pas être liés aux conditions hydriques du milieu. Ces critères, corrélés aux paramètres thermoclimatiques stationnels (Terral et Arnold-Simard 1996; Terral and Mengüal, 1999), représentent une estimation de la vulnérabilité des vaisseaux conduction à l'embolisme gazeux (2) appelée aussi degré de sécurité de conduction. Une densité de vaisseaux élevée chez les oliviers cultivés et irrigués, et un grand nombre de vaisseaux agrégés pour l'olivier sauvage de ripisylve est la parfaite illustration du processus adaptatif suivant. En effet, si la conduction de la sève est désactivée par l'obstruction de vaisseaux, d'autres éléments contigus pourront prendre le relais de la conduction et assurer de ce fait la viabilité des organes innervés.

\section{ARCHÉOLOGIE D'UN SAVOIR-FAIRE ARBORICULTURAL : L'IRRIGATION MÉDIÉVALE DES OLIVIERS}

La construction éco-anatomique du protocole de reconnaissance d'une pratique agraire n'est que la première étape qui conduit à écrire l'histoire des gestes techniques présidant à la culture médiévale de l'olivier. Il faut encore l'appliquer aux charbons de bois archéologiques et, ensuite, comparer au référentiel bâti à partir de l'actuel. Alors seulement la pratique sera réellement documentée techniquement et chronologiquement.

\section{Choix des échantillons traités et contexte des découvertes bioarchéologiques}

Pour que l'étude éco-anatomique soit statistiquement significative, le matériel anthracologique doit être suffisamment fiable : au moins une vingtaine d'échantillons par site sur de faibles populations. Or l'olivier, comme la plupart des arbres fruitiers, n'est pas une espèce prégnante dans les spectres anthracologiques. Cet état de fait, discuté ailleurs (Durand 2004), a conduit à rejeter un certain nombre de sites archéologiques qui n'ont pas livré assez d'échantillons (Lastours, Psalmodi, Plaissan...). Seuls Lunel-Viel (IX ${ }^{\mathrm{e}}$-tout début $\mathrm{XI}^{\mathrm{e}}$ siècle) et l'Ortolo $\left(\mathrm{XV}^{\mathrm{e}}\right.$ siècle), où de nombreux charbons de bois d'Olea europaea ont été identifiés, répondaient à ces conditions. Mais pour apprécier la diffusion géographique et historique d'un savoir-faire arboricultural, deux lots de charbons, presque aux antipodes du millénaire médiéval chacun, n'ont pas paru satisfaisants. L'aire géographique primitive a été élargie à la Catalogne. Les opportunités d'étude ont guidé cette décision. En effet, le site de fours de potiers de Cabrera d'Anoia (XI'-début $\mathrm{XIV}^{\mathrm{e}}$ siècle) étudié de concert par V. Py et A. Durand a livré des échantillons d'oliviers assez abondants. Le site daté du $\mathrm{XV}^{\mathrm{e}}$ siècle de la Antigua Audiencia de Tarragone analysé par E. Allué, préhistorienne de l'université de Tarragone, aussi (3). Malgré des hiatus dans la chronologie et l'éloignement temporel et géographique, mais non bioclimatique, des sites, le matériel rassemblé (4) a semblé suffisant pour être potentiellement exploité. Nous souhaitons qu'il s'enrichisse au fil des années.

Les charbons de bois passés au crible des protocoles éco-anatomiques ne proviennent pas tous des mêmes types de dépôts : c'est un facteur à garder à l'esprit au moment d'interpréter les résultats. Ceux de Lunel-Viel ont été retrouvés dans le comblement limoneux du fossé entourant l'église et ayant fonctionné comme dépotoir. Ces échantillons sont les seuls à avoir été prélevés par un tamisage de 100 litres de sédiment. Aménagé vers les VIII $^{\mathrm{e}}-\mathrm{IX}^{\mathrm{e}}$ siècles, ce fossé a fonctionné durant une très courte période, probablement moins d'un siècle, peutêtre quelques décennies seulement (Raynaud 1989). En effet, les observations de P. Poupet mettent en évidence l'alternance de phases de drainage intense, de

(2) Embolisme gazeux ou cavitation : processus par lequel les vaisseaux conducteurs de sève brute sont obstrués par des bulles d'air. Se référer à Carlquist et Debuhr (1977) et Carlquist (1988).

(3) Nous remercions très vivement E. Allué qui nous a confié les échantillons médiévaux d'oliviers qu'elle a déterminés afin qu'ils puissent être traités dans l'optique méthodologique présentée ci-dessus.

(4) Nous remercions G. Giovannangeli, chercheur associé au LAMM (UMR 6572), C. Raynaud, directeur de recherches au CNRS (UMR 154 Lattes), J. Thiriot, directeur de recherches au CNRS (LAMM-UMR 6572) et I. Padilla Lapuente (Université de Barcelone) de nous avoir confié leur matériel anthracologique. 
pédogenèse et d'apport anthropique conduisant à accélérer le processus de comblement (Poupet in Raynaud 1989). L'abondant mobilier mis au jour est antérieur au $\mathrm{X}^{\mathrm{e}}$ siècle. Les échantillons de Cabrera d'Anoia en Catalogne sont les résidus des bois destinés à alimenter les fours de potiers d'une officine de grande ampleur (Padilla Lapuente et al. 1999 ; Thiriot 2004). Le site a fonctionné du $\mathrm{VII}^{\mathrm{e}}$ au $\mathrm{XV}^{\mathrm{e}}$ siècle, avec des datations radiocarbones très resserrées entre les $\mathrm{XI}^{\mathrm{e}}$ et début du $\mathrm{XIV}^{\mathrm{e}}$ siècles (Padilla Lapuente et al. 1999). Bien que l'on ne puisse exclure totalement les bornes ultimes de la fourchette chronologique, les prélèvements anthracologiques de Cabrera remontent probablement au Moyen Âge central. Les charbons de bois de Tarragone sont aussi des charbons de bois artisanaux : ce sont les restes des dernières cuissons d'un four de potiers. Ce four a été édifié dans le premier quart du $X V^{\mathrm{e}}$ siècle et a fonctionné durant toute la seconde moitié du $\mathrm{XV}^{\mathrm{e}}$ siècle (Curulla et al. 2000 ; Piñol 2000). À l'Ortolo, les échantillons appartiennent à différents contextes : une fosse-dépotoir, des sols d'occupation et d'abandon de maisons ou de structures baptisées «cabanes » d'un village déserté à la fin du $\mathrm{XV}^{\mathrm{e}}$ siècle (Giovannangeli 1992-1998, 2000).

Les charbons de bois analysés proviennent de divers contextes : charbons ruraux, charbons proche d'une ville, charbons artisanaux, charbons de foyer domestique. Cependant, dans chacun des cas, l'origine locale est assurée, même pour les charbons de bois artisanaux, et c'est quand même là l'essentiel dans la perspective de cet article. Cependant la question de la représentativité technique et sociale des charbons étudiés en regard de la réalité médiévale est posée : la réponse en termes paléoécologiques a été donnée (Chabal 1997) ; pour l'instant, elle ne peut pas l'être en termes d'archéohistoire des pratiques agraires. La seule affirmation qui s'impose est que les charbons analysés ne sont pas des charbons exceptionnels ou provenant d'individus exceptionnels.

\section{Identifier les charbons de bois pour caractériser une pratique}

Une fois les mesures éco-anatomiques effectuées sur les charbons de bois archéologiques, les résultats ont été comparés aux analyses statistiques de référence en tant qu'échantillons supplémentaires. Ils ont été intégrés à l'analyse multivariée et donc reconnus ensuite pour appartenir à tel ou tel groupe d'oliviers. Cependant, chez l'olivier, les cernes de croissance sont parfaitement visibles sur du bois immature ou des petites branches alors que ce n'est pas le cas du bois mature, c'est-à-dire de bois provenant de branches âgées ou du tronc (fig.1) (Chakroun 1983 ; Terral 2000). Ainsi, sur certains charbons de bois archéologiques, il a été impossible d'observer les cernes de croissance, soit que le fragment carbonisé provienne effectivement d'une charpentière, soit que la trop petite taille ou la mauvaise conservation de l'échantillon ne l'aient pas permis. D'où la mise au point sur l'actuel de deux AFD différentes, l'une intégrant le critère « longueur du cerne » et l'autre non. Dans le cas où les cernes de croissance n'ont pas pu être observés, les échantillons archéologiques n'ont pas été pris en considération dans les traitements globaux. Chaque charbon de bois archéologique a été attribué à une population d'oliviers précise (tabl. 3, fig. 5 et clichés).

La grande majorité des charbons de bois archéologiques a été classée dans le groupe des charbons de bois cultivés. Quelques individus, toutefois, appartiennent au groupe des oliviers sauvages, sans que l'on puisse dire si ces individus sont nés naturellement sauvages ou si ce sont des arbres cultivés retournés à l'état sauvage. Parmi le groupe des oliviers cultivés, la plupart sont des cultivés irrigués. Un nombre non négligeable de charbons de bois n'a pu être rapporté à un groupe spécifique car ils présentaient des caractéristiques anatomiques intermédiaires entre deux groupes.

Cette répartition dans trois groupes différents éclaire l'origine des charbons de bois des quatre dépôts. En effet, une analyse anthracologique classique ne fournit que peu de renseignements sur la provenance de chaque échantillon d'une même espèce : s'il a dix fragments de saule, l'anthracologue ignore, en l'absence de cassures se recollant, s'ils proviennent d'un seul et même individu ou de plusieurs. Les protocoles d'éco-anatomie affinent les données traditionnelles en montrant la variété originelle : au moins trois sujets différents par site ont été caractérisés, ce qui renforce l'interprétation anthracologique classique d'une représentativité des charbons dispersés dans les couches archéologiques (Chabal 1997).

\begin{tabular}{|lcccccccc|}
\hline Sites & Datation & $\begin{array}{c}\text { Nombre total de } \\
\text { charbons de } \\
\text { bois d'olivier } \\
\text { identifiés }\end{array}$ & $\begin{array}{c}\text { Nombre de } \\
\text { charbons de } \\
\text { bois d'olivier } \\
\text { analysés }\end{array}$ & $\begin{array}{c}\text { Sauvage } \\
\text { milieu sec }\end{array}$ & $\begin{array}{c}\text { Sauvage en } \\
\text { ripisylve }\end{array}$ & $\begin{array}{c}\text { Cultivé à } \\
\text { sec }\end{array}$ & $\begin{array}{c}\text { Cultivé } \\
\text { irrigué }\end{array}$ & $\begin{array}{c}\text { Non } \\
\text { classé }\end{array}$ \\
\hline Lunel-Viel & $\mathrm{IX}^{\mathrm{e}}-\mathrm{X}^{\mathrm{e}} \mathrm{s}$. & 90 & 66 & 11 & 0 & 19 & 16 & 20 \\
Cabrera & $\mathrm{XI}^{\mathrm{e}}-\mathrm{XV}^{\mathrm{e}} \mathrm{s}$. & 21 & 20 & 2 & 0 & 4 & 11 & 3 \\
Tarragona & $\mathrm{XV}^{\mathrm{e}} \mathrm{s}$. & 70 & 35 & 1 & 0 & 8 & 24 & 2 \\
Ortolo & $\mathrm{XV}^{\mathrm{e}} \mathrm{s}$. & 59 & 44 & 2 & 0 & 2 & 32 & 8 \\
\hline
\end{tabular}

Tab. 3 : Répartition des charbons de bois archéologiques médiévaux en fonction des groupes de références définis par deux analyses factorielles discriminantes (AFD). (Analyses A. Durand et J.-F. Terral). 

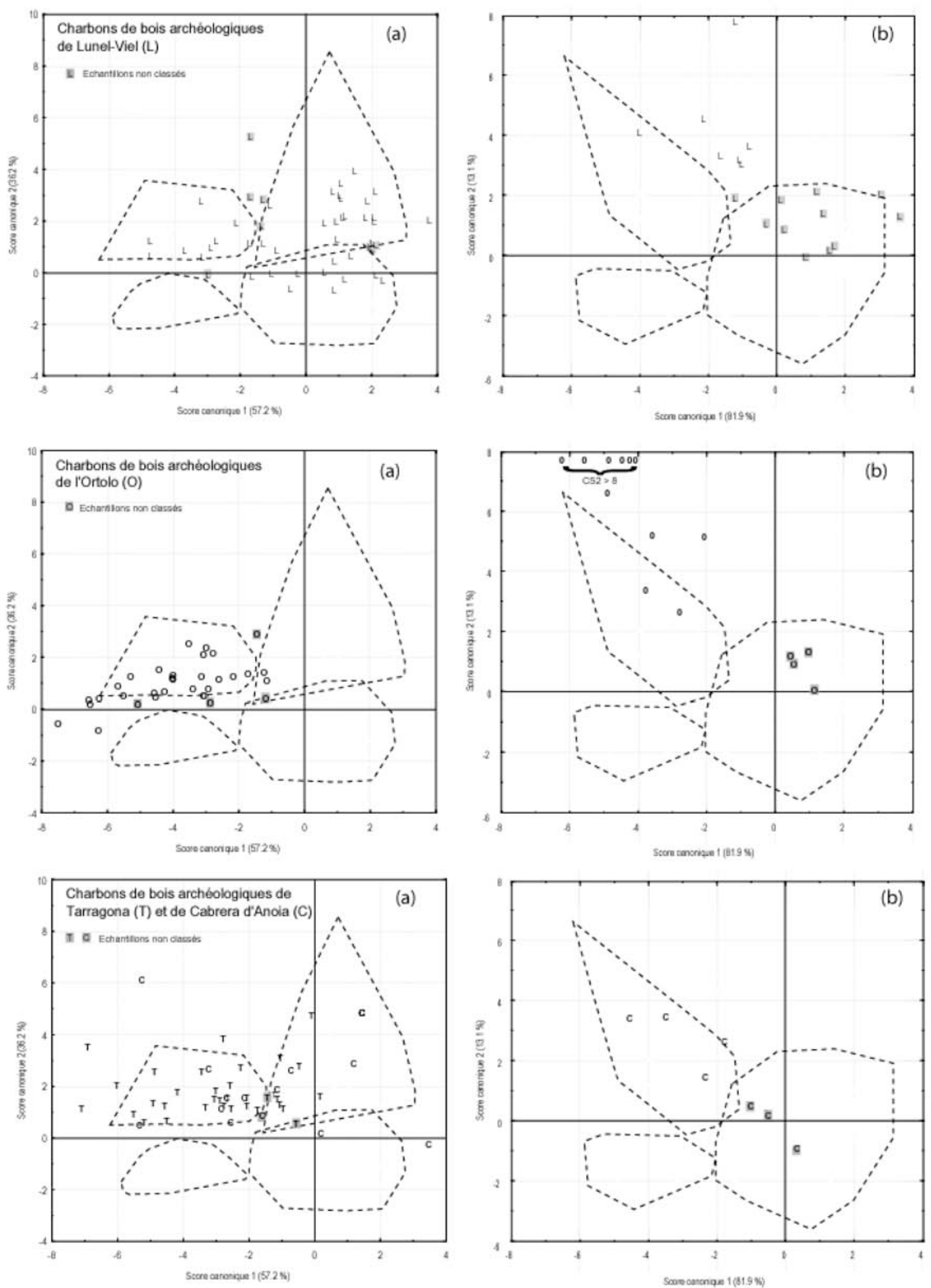

a) AFD pratiquée avec tous les caractères anatomiques b) AFD pratiquée sans le caractère "largeur de cerne"

Fig. 5 : Représentation graphique de la confrontation des données éco-anatomiques issues de l'analyse des charbons de bois archéologiques médiévaux ;

(a) AFD pratiquée sur tous les caractères anatomiques

(b) AFD pratiquée sans le caractère « largeur de cerne ». Analyses A. Durand et J.-F. Terral. 


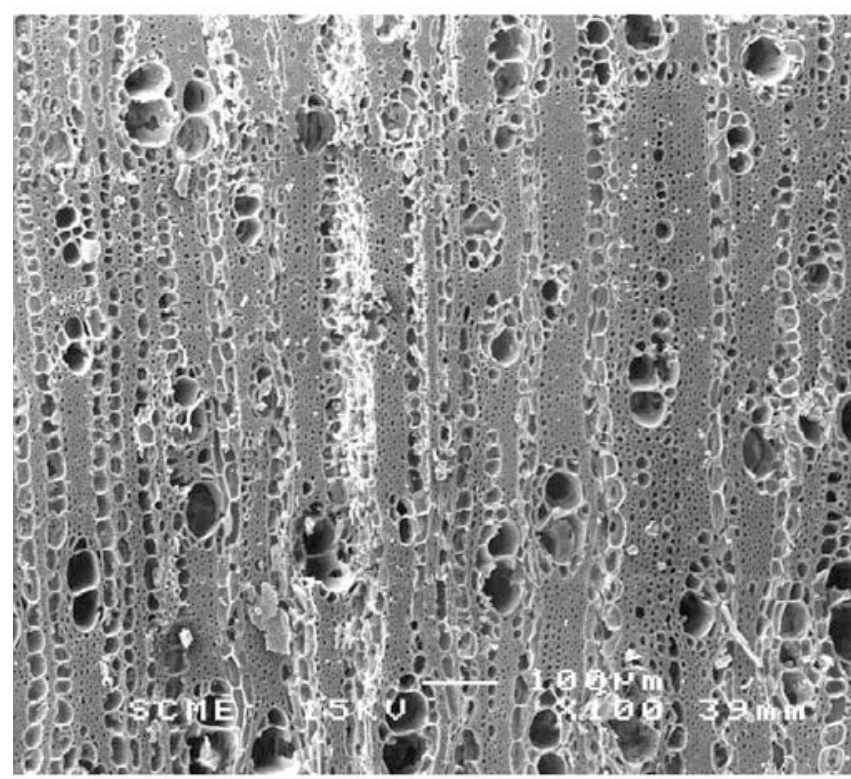

Cliché 1 : Olea europaea irrigué en coupe transversale (x100). Lunel-Viel (IX ${ }^{\mathrm{e}} \mathrm{X}^{\mathrm{e}} \mathrm{s}$.).

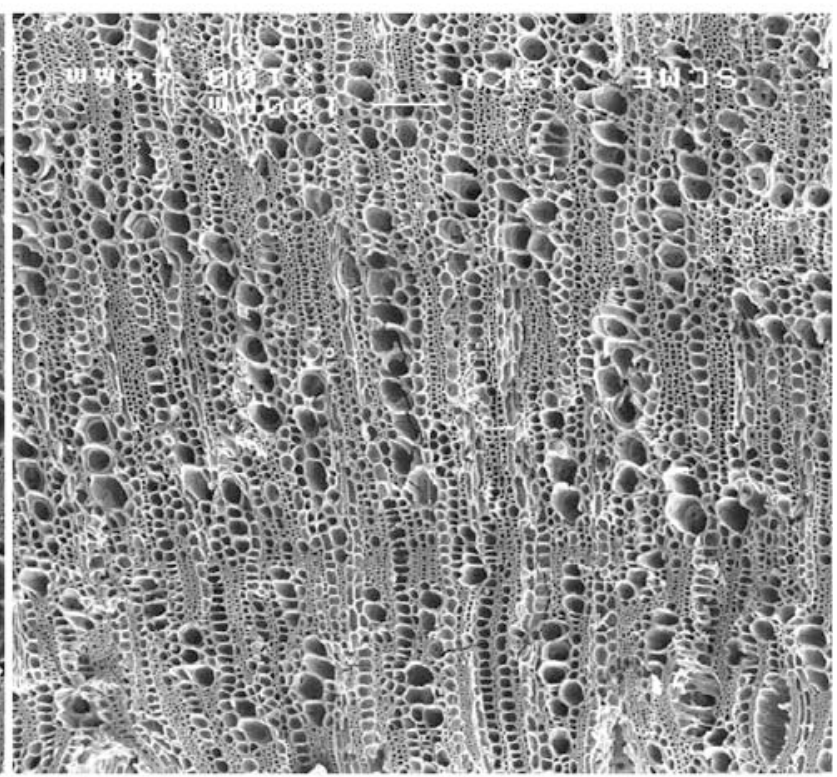

Cliché 2 : Olea europaea irrigué en coupe transversale (x100). Lunel-Viel (IX ${ }^{\mathrm{e}}-\mathrm{X}^{\mathrm{e}} \mathrm{s}$.).

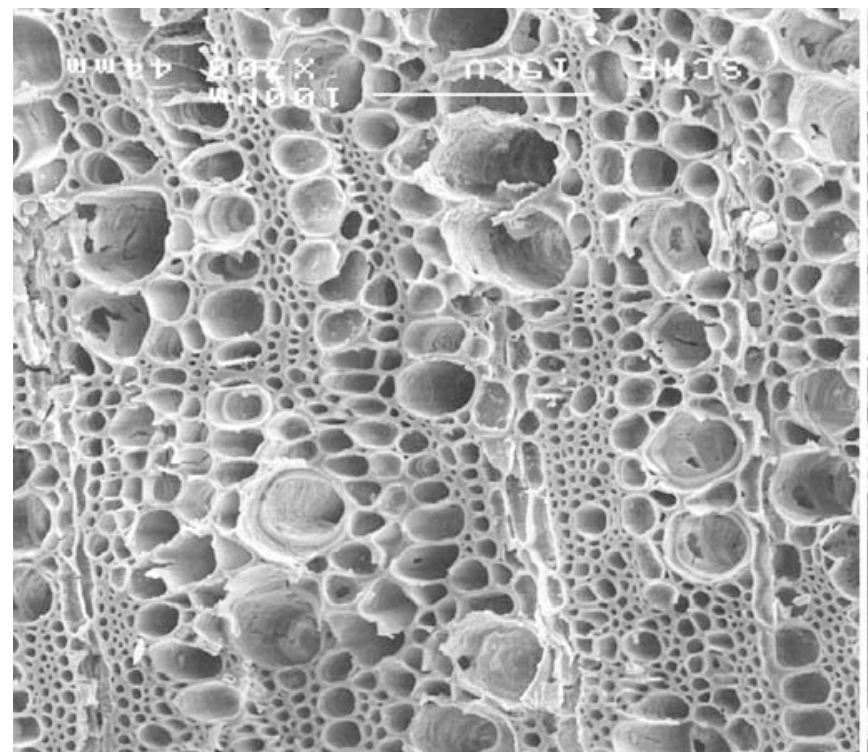

Cliché 3 : Olea europaea irrigué en coupe transversale avec le détail d'une limite de cerne (x300). Lunel-Viel $\left(\mathrm{IX}^{\mathrm{e}}-\mathrm{X}^{\mathrm{e}} \mathrm{s}\right.$.).

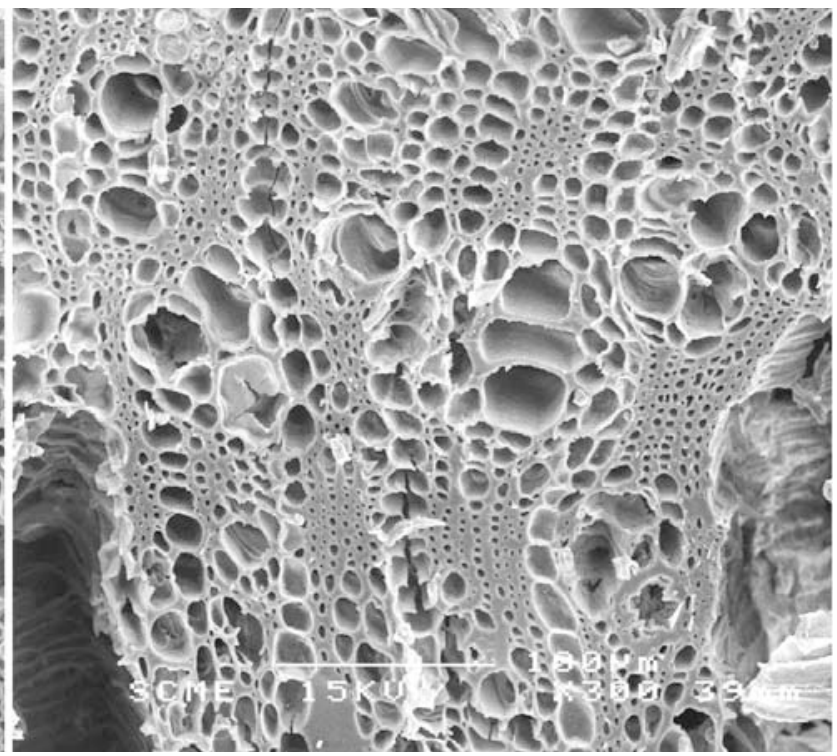

Cliché 4: Olea europaea irrigué en coupe transversale avec détail du groupement des vaisseaux et du parenchyme (x300). Lunel-Viel $\left(\mathrm{IX}^{\mathrm{e}}-\mathrm{X}^{\mathrm{e}}\right)$ 


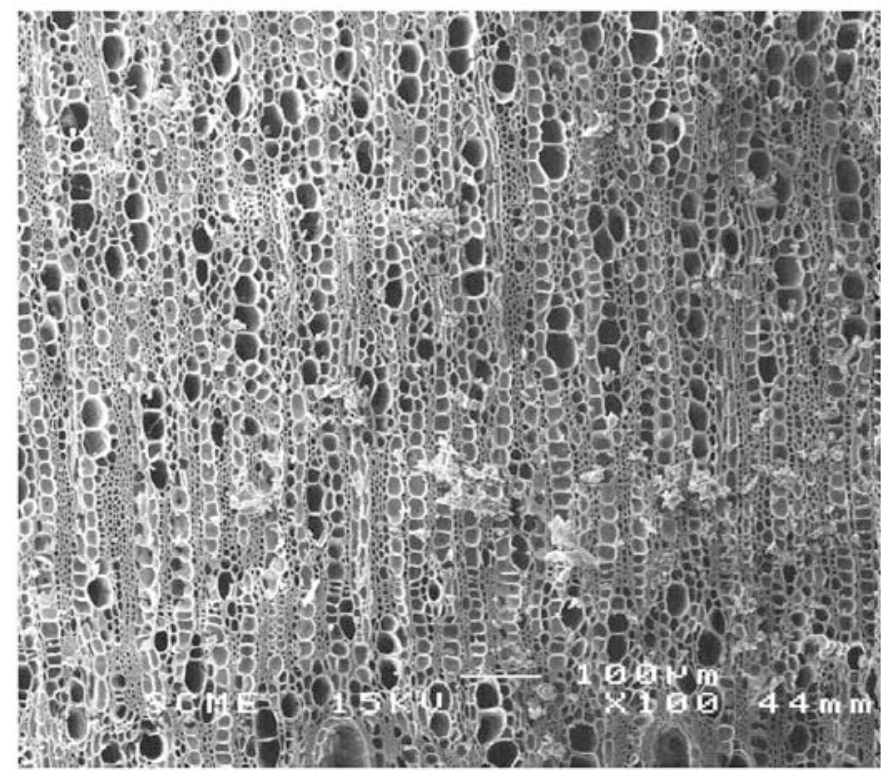

Cliché 5 : Olea europaea irrigué en coupe transversale (x100). Cabrera d'Anoia $\left(\mathrm{XI}^{\mathrm{e}}-\mathrm{XIV}^{\mathrm{e}} \mathrm{s}\right.$. $)$.

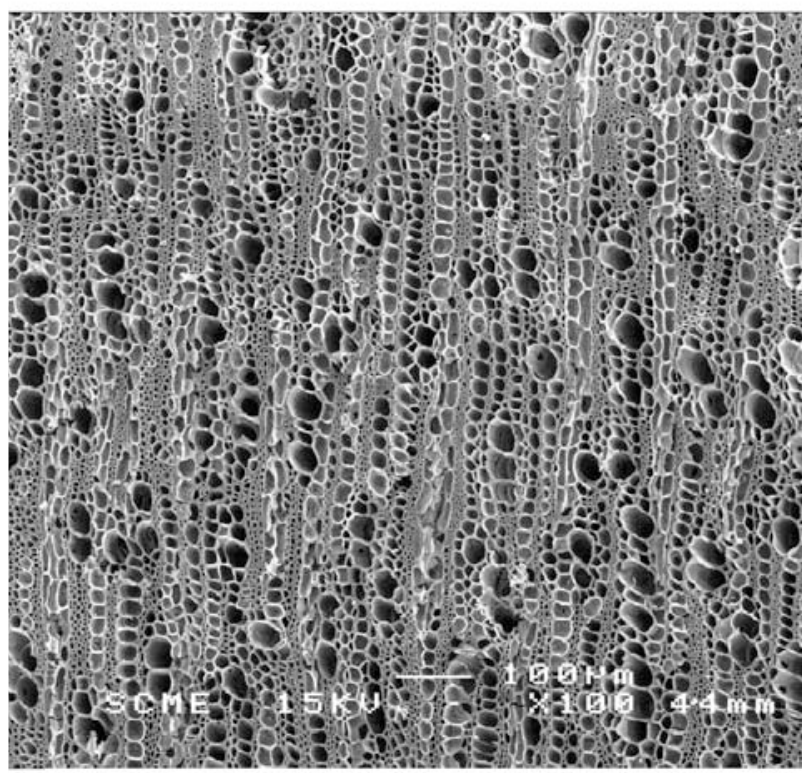

Cliché 6 : Olea europaea irrigué en coupe transversale (x100). Cabrera d'Anoia $\left(\mathrm{XI}^{\mathrm{e}}-\mathrm{XIV}^{\mathrm{e}} \mathrm{s}\right.$.).

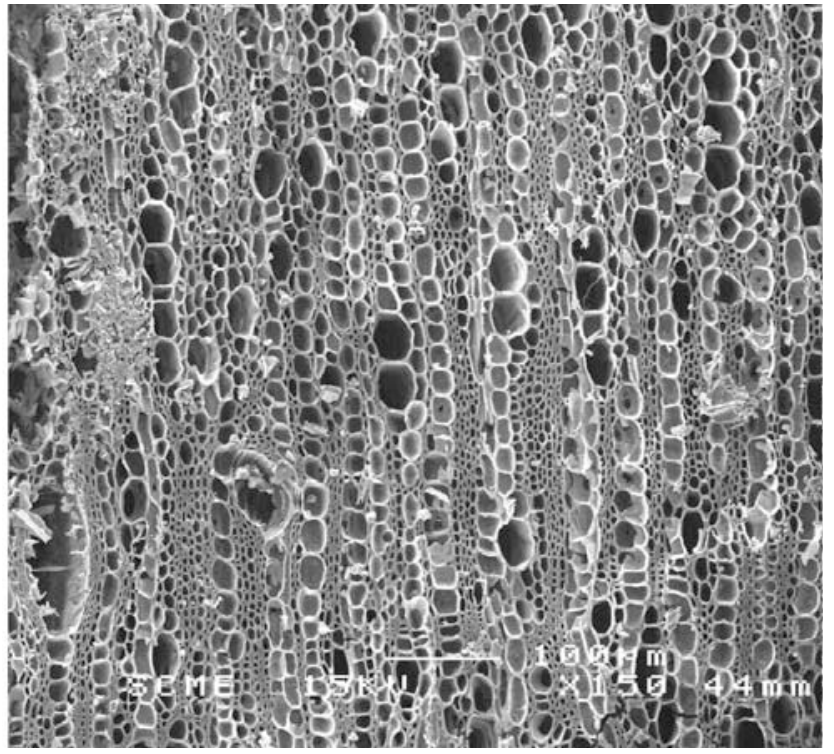

Cliché 7 : Olea europaea irrigué en coupe transversale avec détail du groupement des vaisseaux (x150). Cabrera d'Anoia (XI'-XIV ${ }^{\mathrm{e}} \mathrm{s}$.).

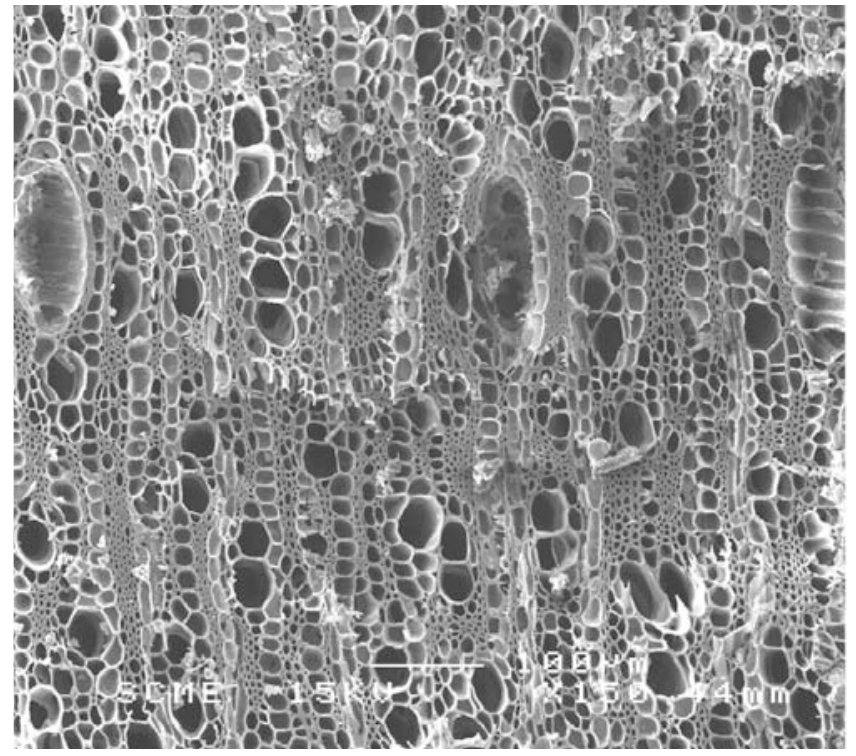

Cliché 8: Olea europaea irrigué en coupe transversale avec détail du groupement des vaisseaux (x150). Cabrera d'Anoia (XI'-XIV ${ }^{\mathrm{e}} \mathrm{s}$.). 


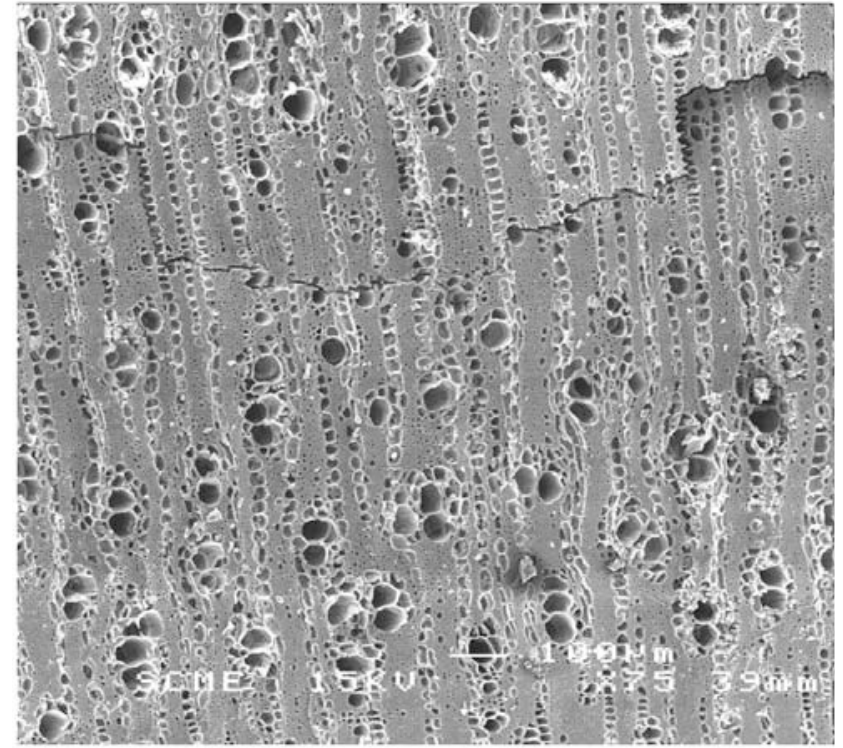

Cliché 9 : Olea europaea irrigué en coupe transversale (x75). L'Ortolo $\left(\mathrm{XV}^{\mathrm{e}} \mathrm{s}\right.$.).

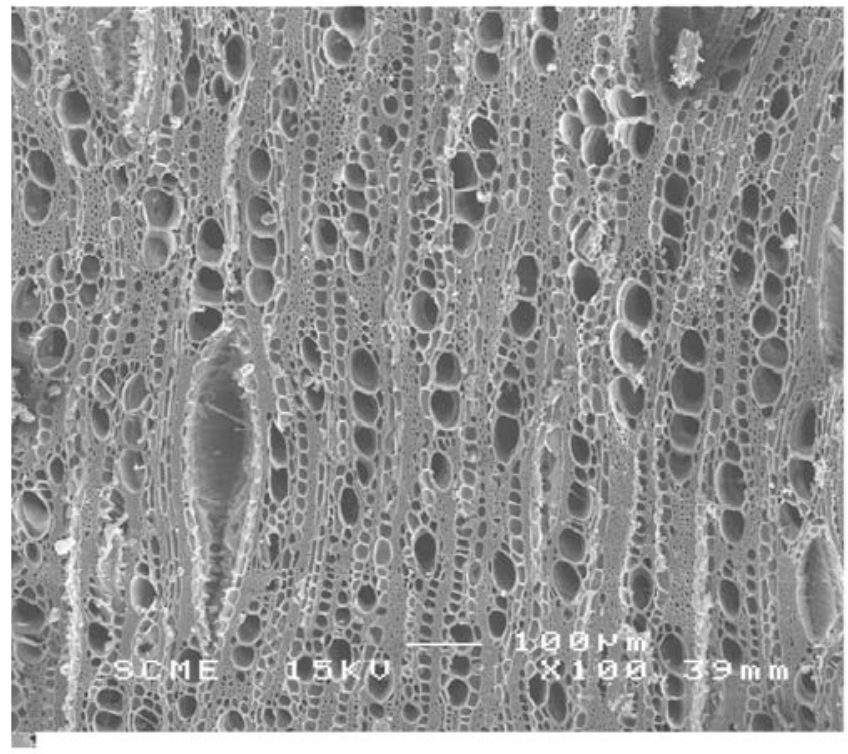

Cliché 10 : Olea europaea irrigué en coupe transversale (x100). L'Ortolo $\left(\mathrm{XV}^{\mathrm{e}} \mathrm{s}\right.$.).

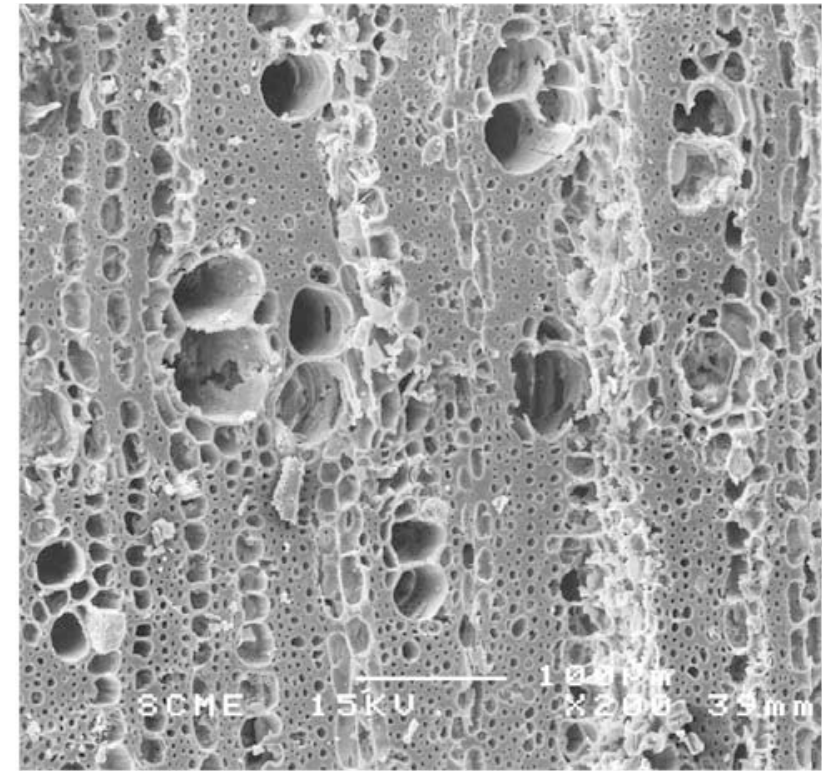

Cliché 11 : Olea europaea irrigué en coupe transversale. avec détail des rayons ligneux et groupement des vaisseaux (x200). L'Ortolo (XV's.).

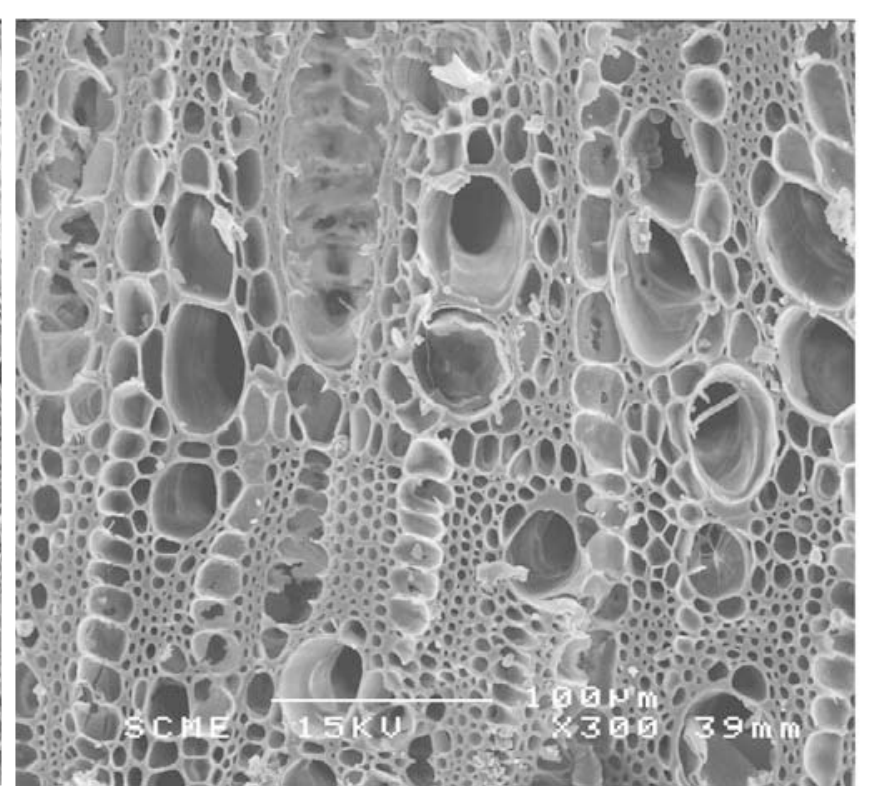

Cliché 12 : Olea europaea irrigué en coupe transversale avec détail du groupement des vaisseaux (x300). L'Ortolo $\left(\mathrm{XV}^{\mathrm{e}} \mathrm{s}\right.$. $)$. 


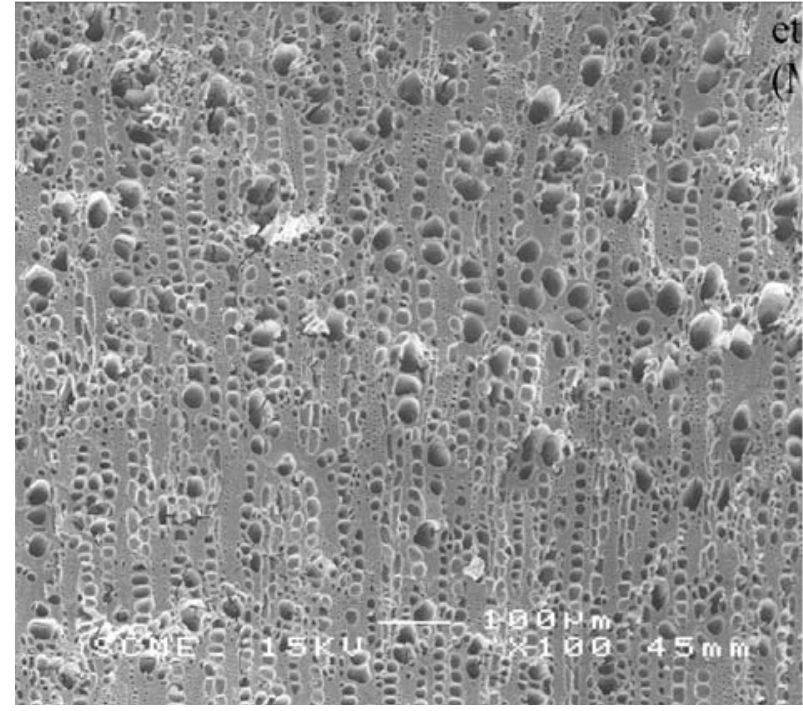

Cliché 13 : Olea europaea ensauvagé en coupe transversale (x 100). Tarragone Antigua Audiencia ( $\mathrm{XV}^{\mathrm{e}} \mathrm{s}$.)

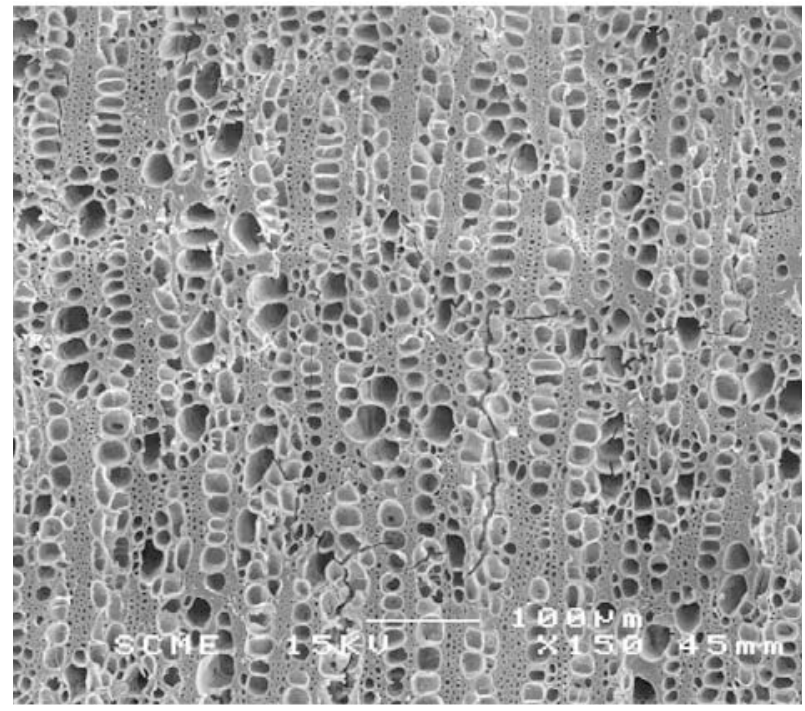

Cliché 14: Olea europaea ensauvagé en coupe transversale (x 150). Tarragone Antigua Audiencia $\left(\mathrm{XV}^{\mathrm{e}} \mathrm{s}\right)$.

N.B : clichés A. Durand, microscope électronique à balayage de l'Université des Sciences et Techniques du Languedoc (Montpellier II).

\section{Reconnaître un savoir-faire}

Les données éco-anatomiques sont révélatrices de la conduite arboriculturale de l'olivier au Moyen Âge : dans trois sites sur quatre, les individus irrigués sont majoritaires, et nettement, sur les individus cultivés à sec. À Lunel-Viel, ils forment une solide minorité. C'est caractériser là un geste technique de manière tangible et directe. La pratique de l'irrigation des olivettes est donc avérée et diffusée dans le monde médiéval. L'anatomie des bois d'oliviers a bel et bien enregistré une pratique : l'apport en eau a été conséquent et régulier ; il ne s'agit pas là de l'effet de pluies plus abondantes, de sols plus humides ou de tout autre cause naturelle ou climatique : le protocole sur l'actuel a intégré ces paramètres et les résultats acquis sur les charbons de bois médiévaux sont indubitablement dûs à un apport d'eau volontaire, à un arrosage récurrent; en aucun cas, les résultats enregistrés sur les charbons de bois archéologiques ne peuvent provenir de cultures d'oliviers faites en zones humides, sur des sols drainés.

Alors que la documentation diplomatique se tait sur ce point, les résultats acquis par l'éco-anatomie quantitative des charbons de bois d'olivier racontent l'histoire d'un geste technique, qui, passé le XII ${ }^{\mathrm{e}}$ siècle, est courant en Méditerranée nord-occidentale. L'usage d'irriguer les olivettes n'est pas une pratique culturale mineure. Elle ne peut être considérée comme un simple palliatif : elle entre sans nul doute dans la chaîne opératoire technique de la culture de l'olivier. C'est qu'il faut accroître le rendement et la qualité de production. Les clichés traditionnellement associés à l'image de l'arbre doivent être sinon déchirés, en tout cas fortement nuancés. La culture médiévale de l'olivier ne doit plus être considérée seulement comme extensive aux marges des terroirs médiévaux, mais aussi comme intensive et parfaitement maîtrisée.

Ces données posent également la question de ce que l'on appelle effectivement irrigation. En effet, les protocoles appliqués ici révèlent un apport d'eau volontaire, régulier, soutenu. Mais comment concrètement ce geste est-il mis en œuvre ? Quelles en sont les modalités pratiques ? Est-ce véritablement une irrigation au sens où les agronomes et les aménageurs modernes et contemporains emploient le mot ? En d'autres termes, comment quantifier cette pratique ? La réponse se trouve sans doute pour une part dans la littérature agronomique susceptible de fournir des exemples de fonctionnement. Elle se trouve sans doute aussi dans l'archéologie du champ et il appartiendra aux praticiens de cette jeune discipline de réinterroger leurs sources, de rechercher de nouvelles méthodologies pour mettre en évidence des traces toujours plus fugaces, toujours plus ténues.

Trop peu de sites sont encore référencés pour que la chronologie de ce savoir-faire puisse être définitive. Lunel-Viel apparaît cependant comme un jalon extrêmement précieux : aux IX $\mathrm{I}^{\mathrm{e}}-\mathrm{X}^{\mathrm{e}}$ siècles, l'irrigation $\mathrm{y}$ est déjà fortement pratiquée, mais pas encore de manière massive. Au contraire, aux $\mathrm{XIII}^{\mathrm{e}}-\mathrm{XV}^{\mathrm{e}}$ siècles, l'irrigation paraît complètement généralisée. Cette mise en ligne 
laisse penser que la pratique d'irriguer les oliviers appartient à cet ensemble de perfectionnements et d'innovations techniques qui portent la croissance agricole du Moyen Âge central. Mais il faudra d'autres analyses pour asseoir définitivement cette conclusion. Tout comme il en faudra de nouvelles pour en apprécier l'origine antique ou médiévale.

\section{CONCLUSION}

L'ambition affichée ici était de renouveler le regard porté par le bioarchéologue, l'archéologue et l'historien sur le charbon de bois archéologique en proposant une autre grille de lecture que la grille de lecture habituellement usitée. La grille usuelle est une grille essentiellement paléoécologique. Celle proposée ici est surtout sociale et technique. Parce que le charbon de bois est aussi un objet culturel qui est façonné de la main de l'homme. Même s'il n'est sans doute pas un artéfact au sens premier du terme, le charbon de bois archéologique porte les stigmates de celui ou de ceux qui l'ont utilisé et mis en oeuvre. Quelque peu négligé jusqu'à présent, le cadre culturel et social doit redevenir une composante légitime de la morphologie anthracologique dans une analyse combinatoire des plans écologique et social qui révèlerait le jeu complexe des différents paramètres et leur mode spécifique d'assemblage. Le protocole mis en œuvre sur l'olivier se résume à quelques pages, quelques tableaux et graphes. Il a pourtant demandé un très lourd investissement de départ qui se compte en années de mises au point et de tests (Terral et Arnold-Simard 1996 ; Terral 1996, 1997a ; Terral et Durand 2006), sans parler de la prise des mesures à proprement parler. C'est pourtant vers ce type de recherches qu'il faudra se tourner à l'avenir.

Depuis un siècle, le divorce entre les sciences de la nature et les sciences de la culture n'a cessé de s'aggraver. Les risques suscités par notre environnement et la peur millénariste portée par l'écologisme de notre société sont à la mesure de l'engouement qui a porté la vague pionnière d'études d'éco-histoire ou d'archéologie des paysages. Cette situation fait trop souvent oublier la dimension culturelle des objets d'étude : le paysage est à la fois une réalité biologique et une construction sociale (Bertrand 1975), le milieu, un milieu de vie pour les humains et le substrat biologique de leur identité (Descola 2001). Envisager un autre regard sur le charbon bois archéologique participe à la compréhension des liens et des interactions complexes entre le champ du social et le champ du biologique. C'est développer là l'étude de faits d'interface, de ces « zones de couplage entre les institutions collectives et les données biologiques et psychologiques qui donnent au social sa substance, mais non sa forme » (Descola 2001). Et dépasser le concept dualiste qui a longtemps sous-tendu et sous-tend encore trop souvent les recherches archéologiques et historiques utilisant des outils paléoenvironnementaux et éco-historiques. Au premier chef, la méthodologie qui vient être exposée participe à la description des objets étudiés en fonction des propriétés que leur attribue : en ce sens, c'est une démarche qui relève de l'anthropologie, et non strictement de l'écologie rétrospective. Elle ouvre la voie à une anthropologie anthracologique des gestes techniques et des pratiques agraires.

\section{REMERCIEMENTS}

Cette contribution a bénéficié du soutien de l'APN jeune chercheur du CNRS (2001-2003) «Éléments pour une histoire de l'arboriculture fruitière au Moyen Âge » (coord. A. Durand, Université de Provence), de l'ACI (2003-2006) «Savoirs en pratique de l'arboriculture fruitière au Moyen Âge : regards croisés sur les techniques de la culture et le corpus fruitier méridional $\left(V^{e}-X V^{e}\right.$ siècle) » (coord. M.-P. Ruas, CNRS) du programme interdisciplinaire du CNRS «Histoire des savoirs» et du GDR «Morphométrie et évolution des formes ».

\section{BIBLIOGRAPHIE}

Amir 1998 : Amir (M.), Les cueillettes de confiance. L'olivier, Alpes de Lumières, t. 129, 1998, p. 203-206.

Amouretti et Comet 1985-2000, AMOURETTI (M.-C.) et COMET (G.), Le livre de l'Olivier, Aix-en-Provence,

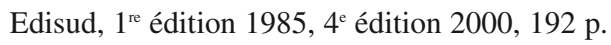

Amouretti et Brun 2002 : AMOURETTI (M.-C.) et BRUN (J.-P.), Oliviers et huile dans l'Antiquité : découvertes archéologiques récentes, in : AMOURETTI (M.-C.) et COMET (G.), éds., Agriculture méditerranéenne, variété des cultures anciennes, Cahiers d'Histoire des Techniques $n^{\circ} 5$, Aix-en-Provence, Presses de l'université de Provence, 2002, p. 127-139.

Baas 1974 : BAAS (J.), Kultur-uns Wildpflanzenreste aus einem römichen Brunnen von Rottweil-Altstadt. In Verdindung mit prähistorischen Schlehenfunden aus Süddeustchland, Fundberichte aus Baden-Württemberg, t. 1, 1974, p. 373-413.

Badal Garcia 1984 : BADAL GARCIA (E.), Contribución al estudio de la vegetación prehistorica del sur de Valencia y norte de Alicante a traves del analisis antracológicos, Thesis de Licenciatura, Universidad de Valencia, 190 p.

Berstch 1941 : BERSTCH (K.), Der Obstbau im vor-und frühgeschichtlichen Deustchland, Germanen-Erbe, t. 6, 1941, p. 103-113.

Berhe 1978 : BEHRE (K. E.), Formenkreise von Prunus domestica L. von der Wikingerzeit bis in die frühe Neuzeit nach Fruchtsteinen aus Haithabu und Alt-Schleswig, $B e$. Deustch. Bot. Ges., t. 91, 1978, p. 161-179.

Behre 1981 : BEHRE (K. E.), The interpretation of anthropogenic indicators pollen diagrams, Pollen et spores, 1981, 23, 2, p. 225-245.

Bertrand 1975 : BERTRAND (G.), Pour une histoire écologique de la France rurale, in : DUBY (G.) et WALLON (G.), dir., Histoire de la France rurale, t. 1, La formation des campagnes françaises des origines à 1340, Paris, Seuil, 1975, p. 34-113. 
Bouby et Marinval 2001 : BOUBY (L.) et MARINVAL (P.), La vigne et les débuts de la viticulture en France : apports de l'archéobotanique, Gallia, t. 58, 2001, p. 143-28.

Boyer 1991 : BOYER (J.-P.), Hommes et communautés du Haut pays niçois médiéval. La Vésubie (XIII $-X V^{e}$ siècle), Nice, Centre d'études médiévales, 1990, 585 p.

Carlquist 1988 : CARLQUIST (S.), Comparative Wood Anatomy. Systematic, Ecological and Evolutionary Aspects of Dicotyledons Wood, Berlin-Heidelberg, Springer-Verlag, $436 \mathrm{p}$.

Chabal 1997 : CHABAL (L.), L'anthracologie, de l'échantillonnage des charbons de bois à l'interprétation $d u$ paysage du Néolithique final à la période romaine en BasLanguedoc, Paris, Documents d'archéologie française, 1997, 189 p.

Chakroun 1983 : CHAKROUN (S.), Les variations saisonnières des formations secondaires (bois, liber, cambium) du tronc de trois espèces méditerranéennes, le Chêne vert, l'Olivier, le Micocoulier: une étude histophysiologique, Thèse de $3^{\text {e }}$ cycle, Université de Montpellier II (Sciences et Techniques du Languedoc), $91 \mathrm{p}$.

Chevalier 1948 : CHEVALIER (A.), L'origine de l'olivier cultivé et ses variations, Revue internationale de botanique appliquée et d'agriculture tropicale, t. 28, 1948, p. 1-25.

Comet 2001 : COMET (G.), L'olivier dans la Provence médiévale : extension et techniques, in : L'olivier dans l'espace et dans le temps, les relations d'un arbre avec son terroir, actes des $1^{\text {res }}$ rencontres internationales de l'olivier tenues à Nyons (19-20 octobre 2000), Nyons, Institut du monde de l'olivier, 2001, p. 31-37.

Comet 2002 : COMET (G.), Le regard d'Olivier de Serres sur l'oléiculture, ou une approche de l'agronomie postmédiévale, in : BELMONT (A.), éd., Autour d'Olivier de Serres. Pratiques agricoles et pensée économique du Néolithique aux enjeux actuels, actes du colloque de Pradel (27-29 sept. 2000), Rennes, Association d'Histoire et sociétés rurales, Bibliothèque d'Histoire rurale, 6, 2002, p. 353-358.

Curulla 2000 : CURULlA (O.), MIR (H.), PIÑOL (L.), El forn de ceràmica medieval de l'antiga Audiència de Tarragona, in : Actes del Ir. Congrés d'archeologia medieval $i$ moderna de Catalunya (13-15 novembre de 1998), Barcelona, ACRAM, 2000, p. 156-166.

Descola 2001 : DESCOLA (Ph.), Leçon inaugurale, Chaire d'anthropologie de la nature, Collège de France, 2001, $35 \mathrm{p}$.

Di Vora et Castelletti 1995 : DI VORA (A.) et CASTELLETTI (L.), Indagine preliminare sull'archeologia della vite (Vitis vinifera L.) in base ai caratteri diagnostici del vinacciolo, Rivista Archeologica dell'Antica Provincia e Diocesi di Como, t. 176, 1995 , p. 333-358.

Durand 1998 : DURAND (A.), Les paysages médiévaux du Languedoc $\left(X^{e}-X I^{e}\right.$ siècles $), \quad$ Toulouse, Presses universitaires du Mirail, 1998, 498 p.

Durand 2004 : DURAND (A.), Du paysage à la pratique, des gestes à l'environnement. Essai d'approche croisée sur les systèmes agraires en France méridionale et en Catalogne
(IX $-X V^{e}$ siècle), Habilitation à diriger les recherches, Université de Provence (Aix-Marseille I), 2 vol., 533 p.

Giovannangeli 1992-1998 : GIOVANNANGELI (G.), Le village d'Ortolo, Sartène (Corse du Sud), Rapports annuels de fouilles programmées, Années 1992-1998, Service régional de l'archéologie de la Corse.

Giovannangeli 2000 : GIOVANNANGELI (G.), Le village d'Ortolo, Sartène (Corse du Sud), Rapport de fouilles programmées, Année 2000, Service régional de l'archéologie de la Corse, 18 p. plus annexe.

Grau Almero 1984 : GRAU ALMERO (E.), El hombre et la vegetación del Neolitico a la Edad del Bronce Valenciano en la Safor (Provincia de Valencia) según la analisisis antracológico de la Cova de la Recambra, Thesis de Licenciatura, Universidad de Valencia, 130 p.

Herrero et Snyder 1997 : HERRERO (J.) and SNYDER (R. L.), Aridity and irrigation in Aragon, Spain, Journal of Arid Environments, t. 35, p. 535-547.

Laval et al. 1990 : LAVAL (H.), LEVEAU (Ph.), MARINVAL (Ph.), MÉDUS (J.), L'olivier et sa culture en Provence. Données historiques, palynologiques et carpologiques, Ecologia Mediterranea, Volume jubilaire en en l'honneur du P. Quézel, t. 16, 1990, p. 427-435.

Leveau et al. 1991 : LEVEAU (Ph.), HEINZ (Ch.), LAVAL (H.), MARINVAL (Ph.) et MÉDUS (J.), Les origines de l'oléiculture en Gaule du Sud. Données historiques, archéologiques et botaniques, Revue d'archéométrie, t. 15, 1991, p. 83-94.

Mangafa et Kostakis 1996 : MANGAFA (M.) et KOSTAKIS (K.), A New Method for the Identification of Wild and Cultivated Charred Grape Seeds, Journal of Archaeological Science, t. 23, 1996, p. 409-418.

Marinval 1988 : MARINVAL (Ph.), Cueillette, agriculture et alimentation végétale de l'Epipaléolithique jusqu'au $2^{e} \hat{A} g e$ $d u \quad F e r$ en France méridionale. Apports paléoethnographiques de la carpologie, Thèse de l'E.H.E.S. S., Paris, 1988, 2 vol., 458 p.

Marinval 1997 : MARINVAL (Ph.), Vigne sauvage et Vigne cultivée dans le Bassin méditerranéen : émergence de la viticulture - Contribution archéo-botanique, in : L'Histoire $d u$ vin, une histoire de rites, Paris, Office International de la Vigne et du Vin, 1997, p. 137-172.

Padilla Lapuente et al. 1999 : PADILLA LAPUENTE (J. I.), THIRIOT (J.), EVIN (J.) et MESTRES (J.), Datation par le radiocarbone des ateliers de potiers médiévaux de Cabrera d'Anoia, in : C14 et Archéologie, Mémoires de la Société de préhistorique française ( $t$. 26), actes $3^{\mathrm{e}}$ congrès international (Lyon 6-10 avril 1998, supplément 1999 de la Revue d'archéométrie, 1999, p. 419-423.

Patumi et al. 2002 : PATUMI (M.), D'ANDREA (R.), MARSILIO (V.), FONTANAZA (G.), MORELLI (G.), Lanza (B.), Olive and olive oil quality after intensive monocome olive growing (Olea europaea L., cv. Kalamata) in different irrigation regimes, Food Chemistry, t. 77, 2002, p. 27-34.

Perlès, 1977 : PERLÈS (C.), La préhistoire du feu, Paris, Masson, 1977, $180 \mathrm{p}$. 
Piñol 2000 : PIÑOL (L.), Baixada del Roser, 1-3 (Antiga audiència), in: CORTÉS (R.), éd., Intervencions arqueológiques a Tarragona i entorn (1993-1999), Barcelone, Servei arqueològic URV, 2000, p. 223-242.

Raynaud 1989, RAYNAUD (C.), Lunel-Viel (Hérault), in : FIXOT (M.) ZADORA-RIO (E.), dir., L'église, le terroir, Paris, C.N.R.S., Monographie du C.R.A. ${ }^{\circ} 1,1989$, p. $105-$ 114.

Renfrew 1973 : RENFREW (J. M.), Palaeoethnobotany. The prehistoric food plants of Near East and Europe, London, Methuen and Co, 1973, 248 p.

Sokal et Rohlf 2000 : SOKAL (R. R.) and ROHLF (J. F.), Biometry : the principles and practices of statistics, in biological research, New York ou San Francisco, 1969, 776 p., Freemann and Co, 1995.

Solari 1988 : Solari (M.-E.), Étude anthracologique des niveaux paléolithique supérieur à l'âge du Bronze de la Cova de l'Espérit (Salses, Pyrénées-Orientales), D.E.A., Université de Montpellier II (Sciences et Techniques du Languedoc, 1988, 56 p.

Stieber 1967 : STIEBER (J.), A Magyaroszgi Felsöpleisztocén vegetáció története az anthrakotómiai eredmények Tükreben, Földtani Közlony, Bulletin of the Hungarian Geological Society, t. 97, 1967, p. 308-317.

Stieber 1969 : STIEBER (J.), A Hasai Késöglacialis Vegetacio történet Anthrakotomiai vizsgalatok Alapjan, Földtani Közlony, Bulletin of the Hungarian Geological Society, t. 99,1969 , p. $188-193$.

Stouff 1988 : STOUFF (L.), L'olivier et l'huile d'olive en Provence aux derniers siècles du Moyen Age, Provence historique, t. 38, 1988, p. 181-191.

Stummer 1911 : STUMMER (A.), Zur Urgeschichte der Rebe und des Weinbaues, Mitteilungen der Anthropologischen Gesellschaft in Wien, t. 6, 1911, p. 283-296.

Terral et Arnold-Simard 1996 : TERRAL (J.-F.) et ARNOLD-SIMARD (G.), Beginnings of Olive tree Cultivation in Eastern Spain in Relation to Holocene Bioclimatic changes, Quaternary Research, 1996, t. 46, p. 176-185.

Terral 1996 : TERRAL (J.-F.), Wild and cultivated olive (Olea europaea L.) : a new approach to an old problem using inorganic analyses of modern wood and archeological charcoal, Review of Paleobotany and Palynology, t. 91, 1996, p. 383-397.

Terral 1997a : TERRAL (J.-F.), La domestication de l'Olivier (Olea europaea L.) en Méditerranée nord-occidentale : approche morphométrique et implications paléoclimatiques. Thèse de l'université de Montpellier II (Sciences et Techniques du Languedoc), 1997, 2 vol., $334 \mathrm{p}$.

Terral 1997b : TERRAL (J.-F.), Débuts de la domestication de l'olivier (Olea europea L.) en Méditerranée nordoccidentale, mise en évidence par l'analyse morphométrique appliquée à du matériel anthracologique, Comptes Rendus à l'Académie des Sciences de Paris, t. 324, série IIa, 1997, p. 417-425.
Terral et Mengüial 1999 : TERRAL (J.-F.) et MENGUÄL (X.), Reconstruction of Holocene climate in southern France and eastern Spain using quantitative anatomy of olive wood and archeological charcoal, Palaeogeography, Palaeoclimatology, Palaeoecology, t. 153, 1999, p. 71-92.

Terral 2000 : TERRAL (J.-F.), Exploitation and Management of the Olive Tree during Prehistoric Times in Mediterranean France and Spain, Journal of Archeological Science, t. 27, 2000, p. 127-133.

Terral et al. 2004 : TERRAL (J.-F.), ALONSO (N.), BUXÒ (R.) I CAPDEVILA, CHATTI (N.), FABRE (L.), FIORENTINO (G.), MARINVAL (P.), PÉREZ JORDA (G.), PRADAT (B.), ROVIRA (N.) et ALIBERT (P.), Historical biogeography of olive domestication (Olea europaea L.) as revealed by geometrical mophometry applied to biological and archaeological material, Journal of Biogeography, t. 31, 2004, p. 1-15.

Terral et Durand, 2006 : TERRAL (J.-F.) et DURAND (A.), Bio-archaeological evidence of olive tree (Olea europaea L.) irrigation during Middle Ages in Southern France and North Eastern Spain, Journal of Archaeological Science, p. 718-724.

Thiébault 1988 : THIÉBAULT (S.), L'homme et le milieu végétal. Analyses anthracologiques de six gisements des Préalpes du Tardi- et au Postglaciaire, Documents d'Archéologie Française, Paris, 1988, 110 p.

Thiriot 2004 : THIRIOT (J.), Les ateliers de potiers médiévaux en Méditerranée : approches archéologiques occidentales, ethnoarchéologie et comparaisons orientales, Habilitation à Diriger les Recherches en archéologie, Université d'AixMarseille I, 3 volumes.

Vernet 1973 : VERNET (J.-L.), Étude sur l'histoire de la végétation du Sud-Est de la France au Quaternaire d'après les charbons de bois principalement, Paléobiologie continentale, 4, 1, 1973, p.1-90.

Vernet 1987 : VERNET (J.-L.), BADAL GARCIA (E.), GRAU ALMERO (E.) et ROS MORA (T.), Charcoal analysis and the western mediterranean prehistoric flora, B.A.R., British Archaeological Reports International Series, vol. 299, 1987, p. 165-178.

Wimmer et al. 2002 : WIMMER (R.), DOWNES (G. M.), EVANS (R.), High-resolution analysis of radial growth and wood density in Eucalyptus nitens, grown under different irrigation regimes, Annals of Forest Sciences, t. 59, 2002, p. 519-524.

Zimmermann 1983 : ZIMMERMANN (M. H.), Xylem structure and the ascent of sap, Berlin-New-York, Springer Verlag, 1983, $143 \mathrm{p}$.

Zohary et Spiegel-Roy 1975 : ZOHARY (D.) et SPIEGELROY (P.), Beginning of fruit growing in the Old Word, Science, t. 187, 1975, p. 319-327.

Zohary et Hopf 1988 : ZOHARY (D.) et HOPF (M.), Domestication of plants in the Old Word, Oxford, Clarenton Press, 1988, 249 p. 